\title{
存在趋势和周期特征的非平稳时间序列的建模 及其应用
}

\author{
王守霞，尤进红，黄涛* \\ 上海财经大学统计与管理学院, 上海 200433 \\ E-mail: wangshouxia20@163.com, johnyou07@163.com, huang.tao@mail.shufe.edu.cn \\ 收稿日期: 2019-09-21；接受日期: 2020-02-20；网络出版日期: 2021-04-06; * 通信作者 \\ 国家自然科学基金 (批准号: 11971291 和 11871323) 和上海财经大学创新团队资助项目
}

\begin{abstract}
摘要 本文研究存在未知周期和趋势的非平稳时间序列的估计问题. 将经典的时间序列分解模型写成 一个含有未知参数的部分线性模型, 首先采用 B- 样条逼近未知时间趋势, 然后利用惩罚最小二乘回 归法得到未知周期、周期序列和趋势的估计. 本文还给出估计量的理论性质, 包括周期估计的相合性 以及周期序列和趋势估计的渐近性质. 模拟研究展现了本文方法的优越性. 最后以两个实际数据为例, 运用本文方法进行建模估计，展现本文方法的实用性.
\end{abstract}

\section{关键词 周期估计 光滑趋势 B- 样条 惩罚最小二乘}

MSC (2020) 主题分类 $62 \mathrm{G} 05,62 \mathrm{G} 08,62 \mathrm{G} 20,62 \mathrm{P} 12$

\section{1 引言}

随着科学研究的不断进步, 对于时间序列的研究方法也在不断完善. 早期人们对于时间序列的研 究主要停留在平稳的时间序列, 而随着时间的推移逐步转移到包含趋势项和周期项的时间序列. 表现 出周期性和趋势性的时间序列广泛存在于现实生活的各个领域, 其中不仅存在周期已知的序列, 如月 度 CPI 数据和季度数据, 而且存在周期未知的序列, 如全球年均气温数据、某地区年降雨量数据和天 文学上太阳黑子的变化数据等.

经典的时间序列分解模型是将序列分解成趋势项、周期项和误差项三部分, 然后运用参数法估计 趋势, 或者采用差分方法剔除周期和趋势. 但是一般而言, 趋势项的具体形式往往是未知的, 如果采用 参数法估计未知趋势, 则需要假定趋势项具有某种函数形式, 如线性形趋势和二次函数趋势等, 一旦 假定错误, 将会对后续建模产生极大的影响. 因此, 参数法存在一定的局限性. 而非参数方法不需要对 未知趋势作某种函数形式的假定, 所以, 非参数方法更为灵活和稳健. 在时间序列研究中可以采用非 参数或者半参数的方法来估计趋势, 如非参数核估计方法或者 B- 样条方法. 
对于周期已知的序列, 人们可以运用季节调整或者季节差分来剔除周期因素. 但是现实生活中还 存在许多周期未知的时间序列, 如气温变化数据、降水量变化数据和天文数据等. 对于周期未知的序 列, 首先要给出一个较为准确的周期估计才能进行后续的研究, 并且周期估计在某些领域十分重要, 如 气候学、天文学和金融等领域. 在气候变化研究中, 我们需要对趋势项和周期项进行精确估算, 这样才 能更加准确地预测未来温度的变化或者其他自然现象出现的时间; 在天文学中, 许多天文现象与我们 的生活息息相关, 例如, 太阳黑子的变化对地球的气候和降水等都会产生很大影响, 而研究表明太阳黑 子的变化是存在周期性的. 此外, 宇宙中存在许多变星, 即亮度有起伏变化的恒星, 变星对科学研究具 有十分重要的意义, 而且对于它们的研究也十分困难. 如果我们能够给出一种较为准确的周期估计方 法, 将会对天文学的研究有很大帮助.

处理时间序列未知趋势的传统方法一般是差分剔除法, 也可以利用参数法估计未知趋势, 如多项 式拟合法和指数平滑法等. 采用参数法估计趋势时, 必须假定趋势项具有某种函数形式. 一旦假定错 误, 得到的结果可能有较大的偏差. 因此, 参数法存在一定的局限性, 不具有非参数方法的灵活性. 随 着非参数统计的发展, 非参数方法也被不断应用于时间序列分析. 文献 [1] 采用非参数核估计方法估 计未知趋势, 文献 [2] 研究如何运用 B- 样条方法估计自回归时间序列的未知趋势, 文献 [3] 运用 B- 样 条方法研究自回归移动平均 (auto-regressive and moving average, ARMA) 模型的有效估计. 与核估计 方法相比, B- 样条方法具有一定的优越性, 原因在于 B- 样条方法的计算更快 (参见文献 [4]).

关于周期已知的时间序列的分析方法已经发展成熟, 例如, 我们可以采用传统的季节差分法进行 季节调整. 对于周期未知的时间序列, 也有大量的文献研究如何对其周期进行估计. 首先发展起来的 周期估计法是参数法. 传统的参数法是基于频域分析的三角函数回归法, 即基于 Fourier 变换利用有 限个三角函数的线性组合来表达周期项, 然后基于周期图或者谱密度去估计未知参数. 文献 [5-8] 都 对这种方法的发展做了大量的研究. 文献 [5] 运用三角函数回归法研究带有独立同分布误差的谐波分 量的估计问题; 文献 [6] 考虑误差的协方差的结构, 运用加权最小二乘法估计频率; 文献 [7] 运用极大 似然估计法对带有 Gauss 误差的时间序列的频率进行研究; 文献 [8] 也对基于周期图的三角函数回归 法作出重要的贡献.

近年来, 非参数周期估计法也在不断发展. 首先是基于时域分析的非参数方法. 对于随机非等距 观测的时间序列, 例如 $\boldsymbol{Y}_{t}=m\left(\boldsymbol{X}_{t}\right)+\varepsilon_{t}$, 此时周期项 $m$ 是一个周期函数, 而本文中 $m$ 是一个周期序 列. 对于这种设定的模型, 很多学者考虑运用核最小二乘法进行估计, 例如, 文献 [9] 在误差项为独立 同分布的假设下, 对周期函数不施加任何参数限制, 直接运用非参数核最小二乘回归法估计序列的周 期. 此外, 现实生活中存在含有多个周期的时间序列, 文献 [10] 运用非参数核最小二乘回归法对多周 期的问题进行了研究. 有些时间序列中的周期会随着时间的变化而变化, 这样的数据在天文学中较多, 如长蛇座 $\mathrm{R}$ 的星等变化周期会随着时间缓慢变化. 受这个数据的启发, 文献 [11] 对变周期的序列进 行了研究, 先假定周期及其变化幅度随着某个参数函数的变化而变化, 然后基于非参数核估计得到周 期参数及周期函数的估计. 除此之外, 还有基于频域分析的非参数周期估计的方法, 例如, 文献 [12] 采 用非参数周期图法对单周期和多周期问题进行了研究.

对于等距观测的时间序列, 周期项 $m$ 是一个周期序列, 而不是前面提到的周期函数. 文献 [13] 将 周期估计问题看成模型选择问题, 运用交叉验证的方法估计未知周期, 并且在文中比较了 Akaike 信息 准则 (Akaike information criterion, AIC)、Bayes 信息准则 (Bayesian information criterion, BIC) 和交 叉验证方法在估计未知周期方面的优缺点. 由 BIC 方法得到的周期估计具有相合性, 而通过 AIC 和 交叉验证法得到的周期估计不是相合估计. 前面的文献都是对只存在周期分量和独立误差的序列进行 研究. 但是时间序列往往会存在趋势分量, 并且时间趋势的存在也会影响周期的估计. 文献 [14] 对存 
在光滑趋势的周期序列进行了研究, 运用惩罚最小二乘法和局部线性核估计法分别估计周期和趋势.

但是, Vogt 和 Linton ${ }^{[14]}$ 的方法 (在本文中简称为 V\&L) 存在一定的局限性, 原因在于他们在估 计周期时直接忽略趋势项, 这意味着将 $\varepsilon+g$ 作为新的误差项 $\varepsilon^{*}$, 当趋势变化较小时, $\varepsilon^{*}$ 与原始误差 $\varepsilon$ 相差不大; 如果趋势 $g$ 的波动变化较大, 那么忽略趋势项会对周期估计产生很大影响, 也会增大估计 的方差. 如图 1 所示, 假设误差来自标准正态分布, 左边趋势项变化较小, 新的误差基本仍旧在 \pm 2 之 间; 但当趋势变化较大如变为原来的 6 倍时, 明显地, 新的误差尽管期望为 0 , 但方差增大了很多, 导 致这个部分占据主导地位, 周期项 $m$ 不再明显, 所以可能难以选择出正确的周期.

为了克服这个局限性, 本文改进了 V\&L, 首先利用 B- 样条逼近未知趋势, 然后运用惩罚最小二乘 估计法估计序列的周期和趋势. 为了更清晰地展现趋势波动对周期估计的影响, 我们给出了逼近趋势 和忽略趋势后的残差平方和随趋势的变化而变化的图 (图 2), 趋势项 $g(u)=K u^{2}-K / 3, u \in[0,1]$, 其 他设定与第 4 节的模拟一相同.

我们可以看到, 在趋势变化较小时两种方法的残差平方和几乎相等, 并且本文利用样条逼近趋势 后的 $\frac{1}{T} \mathrm{RSS}$ 的均值和标准差基本不会随着趋势幅度的变化而变化. 但是忽略趋势项后的 $\frac{1}{T} \mathrm{RSS}$ 的均 值和标准差随着趋势变化幅度的增大而增大. 所以, 误差项会在趋势变化较大时占据主导地位, 周期项 $m$ 不再明显, 从而导致直接忽略趋势可能难以选择出正确的周期, 这也是我们首先利用样条逼近趋势 的原因.

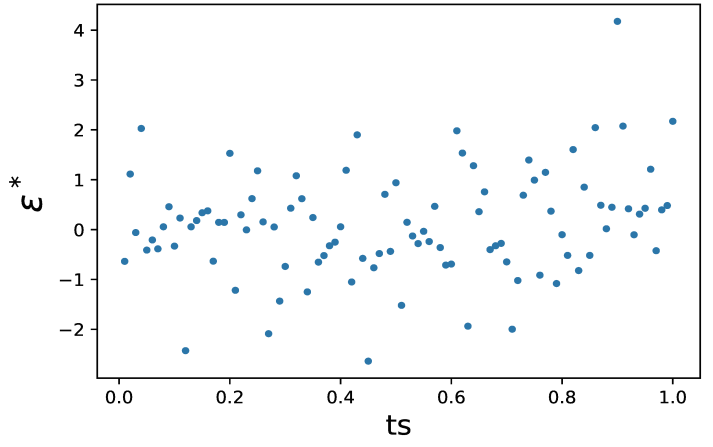

(a)

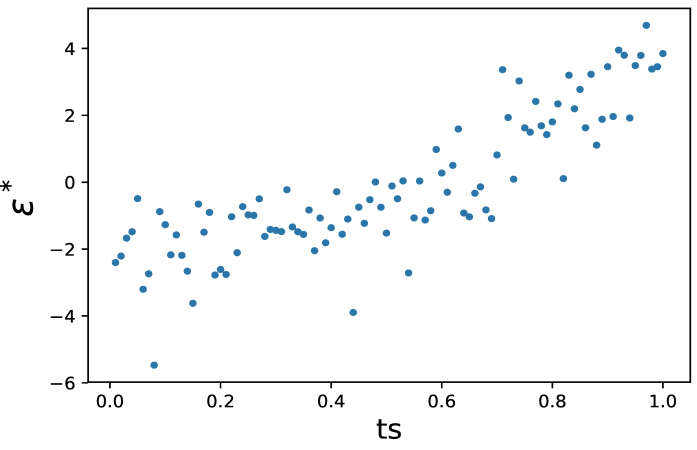

(b)

图 1 (网络版彩图) $\varepsilon \sim N(0,1)$ 时, $\varepsilon^{*}$ 的分布. (a) $g=u^{2}-1 / 3$; (b) $g=6 u^{2}-2$

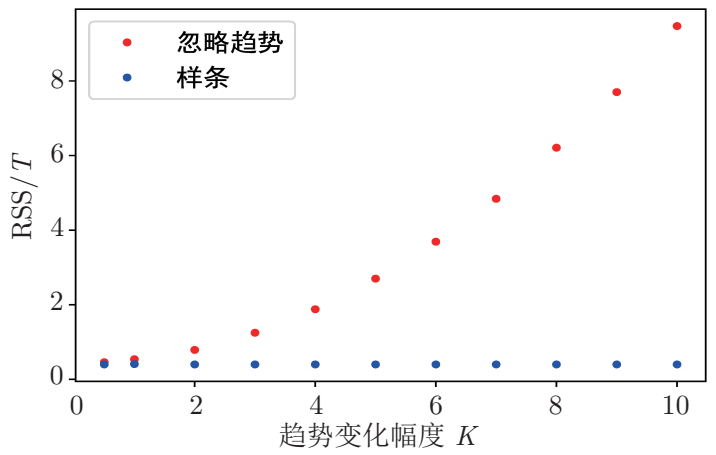

(a)

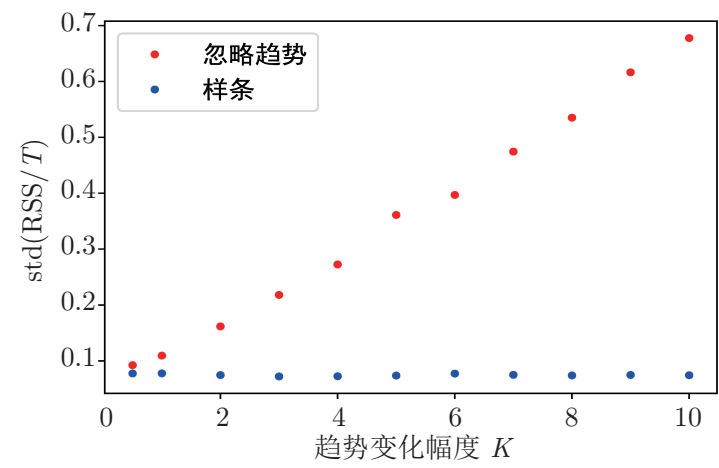

(b)

图 2 (网络版彩图) $T=100, \sigma^{2}=0.5$, 真实周期 $\theta_{0}=12$, 本文方法和 $V \& L$ 在不同趋势函数变化幅度 $K$ 下 的 RSS/T 的均值和标准差. (a) RSS/T 的均值的变化; (b) RSS/T 的标准差的变化 
本文余下内容的安排如下: 第 2 节介绍主要模型, 将带有周期项和趋势项的时间序列写成半参数 模型, 接下来运用 B- 样条逼近未知趋势函数, 然后利用惩罚最小二乘估计法估计周期、周期序列和趋 势. 第 3 节给出估计的理论性质, 包括周期估计 $\hat{\theta}$ 的相合性、周期序列估计 $\hat{m}$ 及趋势估计 $\hat{g}$ 的渐近 分布. 第 4 节通过模拟结果说明本文改进的方法的优点. 第 5 节将本文的方法应用于实际数据. 第 6 节总结本文方法的优缺点并展望未来可能的研究方向. 附录 $\mathrm{A}$ 给出本文定理的证明.

\section{2 模型及估计方法}

经典的时间序列分解方法是将时间序列写成周期项、时间趋势项和噪声的和, 即

$$
Y_{t, T}=g\left(\frac{t}{T}\right)+m(t)+\varepsilon_{t, T}, \quad t=1, \ldots, T,
$$

其中 $\mathrm{E}\left[\varepsilon_{t, T}\right]=0$, 本文中不假定 $\left\{\varepsilon_{t, T}\right\}$ 独立, $\left\{\varepsilon_{t, T}\right\}$ 可以是短期相关的, 例如, $\left\{\varepsilon_{t, T}\right\}$ 可以来自一阶自 回归 $(\mathrm{AR}(1))$ 模型; $g$ 是趋势函数, $\{m(t)\}_{t \in \mathbb{Z}}$ 是周期为 $\theta_{0}$ 的周期序列 ( $\theta_{0}$ 是一个未知整数). 为了保 证模型的可识别性, 假定 $\mathrm{E}\left[g\left(\frac{t}{T}\right)\right]=0$.

需要特别说明, $\{m(t)\}_{t \in \mathbb{Z}}$ 是周期序列而不是函数, 文献 [14] 给出了这个假设的原因. 对于等距观 测的时间序列, 在每个时间点 $t$, 存在很多函数值为 $m(t)$ 的函数, 因此难以识别出正确的周期函数. 此 外, 注意到周期项 $m$ 是定义在真实时间 $t$ 上的序列, 这样可以方便研究周期序列估计的渐近性质, 原 因在于, 随着样本量 $T$ 的增加, 整个周期序列中与 $m(s)$ 相等的样本点也会增加, 我们会获得更多关于 $m(s)$ 的信息. 而趋势项 $g$ 的定义则与周期项 $m$ 相反, 趋势项 $g$ 是定义在单位化时间 $\frac{t}{T}$ 上的函数, 这 种单位化时间的定义在很多文献中也十分常见. 例如, 文献 [15] 也采用了这种单位化的时间, 这样做 可以方便研究 $g$ 的渐近性质. 随着样本量 $T$ 的增加, 在趋势函数的每个小区间内的样本点也越来越 多, 因而可以更容易得到估计的性质, 如相合性、渐近正态性和有效性等.

前面提到 $\{m(t)\}_{t \in \mathbb{Z}}$ 是一个周期为 $\theta_{0}$ 的周期序列, 我们可以通过改写 $\{m(t)\}$ 来改写模型 $(2.1)$. 令 $\boldsymbol{\beta}=\left(\beta_{1}, \ldots, \beta_{\theta_{0}}\right)^{\mathrm{T}}=\left(m(1), \ldots, m\left(\theta_{0}\right)\right)^{\mathrm{T}}$, 则 $\{m(t)\}=\sum_{s=1}^{\theta_{0}} \beta_{s} I_{s}(t)$, 其中 $I(\cdot)$ 是一个示性函数, $I_{s}(t)=I\left(t=k \theta_{0}+s\right.$ 对某个 $k$ 成立, $\left.k \in \mathbb{N}\right)$. 所以, 模型 (2.1) 可以改写成如下形式:

$$
Y_{t, T}=g\left(\frac{t}{T}\right)+\sum_{s=1}^{\theta_{0}} \beta_{s} I_{s}(t)+\varepsilon_{t, T}, \quad t=1, \ldots, T .
$$

模型 (2.2) 的矩阵形式如下:

$$
\boldsymbol{Y}=\boldsymbol{g}+\boldsymbol{X}_{\theta_{0}} \boldsymbol{\beta}+\varepsilon
$$

其中 $\boldsymbol{Y}=\left(Y_{1, T}, \ldots, Y_{T, T}\right)^{\mathrm{T}}$ 是由观测值组成的向量, $\boldsymbol{g}=\left(g\left(\frac{1}{T}\right), \ldots, g\left(\frac{T}{T}\right)\right)^{\mathrm{T}}$ 是趋势项, $\boldsymbol{\varepsilon}=\left(\varepsilon_{1, T}, \ldots\right.$, $\left.\varepsilon_{T, T}\right)^{\mathrm{T}}$ 是误差向量, $\boldsymbol{X}_{\theta_{0}}=\left(\boldsymbol{I}_{\theta_{0}}, \boldsymbol{I}_{\theta_{0}}, \ldots\right)^{\mathrm{T}}$ 是 $T \times \theta_{0}$ 的设计矩阵, $\boldsymbol{I}_{\theta_{0}}$ 是 $\theta_{0} \times \theta_{0}$ 的单位矩阵. 由于模型 (2.3) 线性部分的设计矩阵 $\boldsymbol{X}_{\theta_{0}}$ 存在未知参数 $\theta_{0}$, 所以模型 (2.3) 不是一个普通的半参数部分线性模 型. 但是, 如果周期是已知的, 那么模型 (2.3) 退化成一个半参数部分线性模型, 我们可以很容易得到 周期序列 $m$ 以及趋势 $g$ 的估计. 因此接下来需要先对未知周期 $\theta_{0}$ 进行估计.

下面主要讨论如何估计未知周期 $\theta_{0}$ 、周期序列 $m$ 和趋势 $g$. 前面已经说明本文在估计未知周期 
时没有忽略趋势项, 而是运用 B- 样条逼近趋势 $g$. B- 样条在估计未知函数方面是个非常实用的工具, 被广泛地应用于变系数模型和可加模型等模型的估计.

\subsection{B- 样条}

假设 $r$ 是一个正整数, 存在一系列等距分布的节点 $\tau:=\left\{\tau_{j}\right\}_{j=1}^{N}$ 将区间 $[0,1]$ 等分成 $N+1$ 个小 区间, $J_{j}=\left[\tau_{j}, \tau_{j+1}\right), j=0, \ldots, N-1, J_{N}=\left[\tau_{N}, 1\right]$, 其中 $\tau:=\left\{\tau_{j}\right\}_{j=1}^{N}$ 叫作内节点; 给定

$$
\tau_{1-r}=\cdots=\tau_{-1}=\tau_{0}=0<\tau_{1}<\cdots<\tau_{N}<1=\tau_{N+1}=\cdots=\tau_{N+r},
$$

其中 $\tau_{j}=j h, j=0,1, \ldots, N+1, h=1 /(N+1)$ 是相邻两个节点之间的距离. $\mathcal{H}_{N}^{r-2}:=\mathcal{H}_{N}^{r-2}[0,1]$ 表示 一个函数空间, 对任意函数 $g \in \mathcal{H}_{N}^{r-2}, g$ 在每个小区间 $J_{j}$ 上是一个 $r-1$ 次的多项式, 并且有 $r-2$ 阶 连续导数. 那么 $\mathcal{H}_{N}^{r-2}$ 上的一组 B- 样条基函数是 $\left\{b_{j, r}(u), j=1-r, \ldots, N\right\}$, 其中 $b_{j, r}$ 表示第 $j$ 个 $r$ 阶 B- 样条基函数. 当 $r=1$ 时,

$$
b_{j, 1}(u)= \begin{cases}1, & u \in J_{j}, \\ 0, & \text { 其他. }\end{cases}
$$

$b_{j, r}$ 可以由文献 [16] 中的递归算法给出:

$$
b_{j, r+1}(u)=\frac{u-\tau_{j}}{\tau_{j+r}-\tau_{j}} b_{j, r}(u)+\frac{\tau_{j+r+1}-u}{\tau_{j+r+1}-\tau_{j+1}} b_{j+1, r}(u) .
$$

对于本文的时间序列 $\boldsymbol{Y}$, 定义

$$
\boldsymbol{b}_{j}=\left(b_{j, r}\left(u_{1}\right), \ldots, b_{j, r}\left(u_{T}\right)\right)^{\mathrm{T}}, \quad \boldsymbol{B}\left(u_{t}\right)=\left(b_{1-r, r}\left(u_{t}\right), \ldots, b_{N, r}\left(u_{t}\right)\right)^{\mathrm{T}},
$$

其中 $u_{t}=t / T, t=1, \ldots, T ; \boldsymbol{B}$ 是 B- 样条基函数在样本点处取值得到的 $T \times(N+r)$ 矩阵,

$$
\boldsymbol{B}=\left(\boldsymbol{b}_{1-r}, \ldots, \boldsymbol{b}_{N}\right)=\left(\boldsymbol{B}\left(u_{1}\right), \ldots, \boldsymbol{B}\left(u_{T}\right)\right)^{\mathrm{T}}
$$

注意到, B- 样条基函数的个数与节点数和基函数的阶数有关, 严格来说基函数的个数 $N_{T}$ 等于内部节 点数 $N$ 加上基函数的阶数 $r$. 所以, 趋势函数 $\boldsymbol{g}$ 可以用 $\boldsymbol{B} \boldsymbol{\alpha}$ 估计, 其中 $\boldsymbol{B}=\left(\boldsymbol{b}_{1}, \ldots, \boldsymbol{b}_{N_{T}}\right)(t=1, \ldots, T)$ 是 $T \times N_{T}$ 的矩阵, $N_{T}=N+r$, 与前面给出的符号相同, $\boldsymbol{b}_{j}=\left(b_{j, r}\left(\frac{1}{T}\right), \ldots, b_{j, r}\left(\frac{T}{T}\right)\right)^{\mathrm{T}}$ 是由第 $j$ 个 $r$ 阶 B- 样条基函数在所有单位化时间点 $\frac{t}{T}$ 处的取值构成的向量, $\alpha$ 是 $N_{T} \times 1$ 的向量. 那么模型 (2.3) 可 以写成如下形式:

$$
\boldsymbol{Y}=\boldsymbol{B} \boldsymbol{\alpha}+\boldsymbol{X}_{\theta_{0}} \boldsymbol{\beta}+\varepsilon+\boldsymbol{g}-\boldsymbol{B} \boldsymbol{\alpha}=\boldsymbol{Z}_{\theta_{0}} \gamma+\boldsymbol{\varsigma}
$$

其中 $\boldsymbol{Z}_{\theta_{0}}=\left(\boldsymbol{X}_{\theta_{0}}, \boldsymbol{B}\right), \boldsymbol{\gamma}^{\mathrm{T}}=\left(\boldsymbol{\beta}^{\mathrm{T}}, \boldsymbol{\alpha}^{\mathrm{T}}\right), \boldsymbol{\varsigma}=\boldsymbol{\varepsilon}+\boldsymbol{g}-\boldsymbol{B} \boldsymbol{\alpha}$.

\section{2 未知周期 $\theta_{0}$ 的估计}

对于未知周期的估计, 我们可以将这个问题看成模型选择问题, 那么一个很自然的想法是, 运用最 小二乘回归法, 通过最小化残差平方和得到周期估计. 具体而言, 对每个可能的周期 $\theta, \theta \in\left[1, \Theta_{T}\right]$, 我 
们对模型 (2.4) 作最小二乘回归, 得到一个残差平方和, 然后选择使得残差平方和达到最小的周期 $\theta$. 这里 $\Theta_{T}$ 是所有可能的周期备选值 $\theta$ 的上界, 并且 $\Theta_{T}$ 可以随着样本量的增加而增大, 第 3 节给出其 具体说明. 对每个备选的周期 $\theta$, 我们可以很容易得到 $\gamma_{\theta}$ 的最小二乘估计 $\hat{\gamma}_{\theta}=\left(\boldsymbol{Z}_{\theta}^{\mathrm{T}} \boldsymbol{Z}_{\theta}\right)^{-1} \boldsymbol{Z}_{\theta}^{\mathrm{T}} \boldsymbol{Y}$, 其中 $\boldsymbol{Z}_{\theta}=\left(\boldsymbol{X}_{\theta}, \boldsymbol{B}\right), \boldsymbol{X}_{\theta}=\left(\boldsymbol{I}_{\theta}, \boldsymbol{I}_{\theta}, \ldots\right)^{\mathrm{T}}$ 是 $T \times \theta$ 的设计矩阵, $\boldsymbol{I}_{\theta}$ 是 $\theta \times \theta$ 的单位矩阵. 相应地, 我们可以计 算出每一个备选周期 $\theta$ 对应的残差平方和, 记为 $\operatorname{RSS}(\theta)$ :

$$
\operatorname{RSS}(\theta)=\left\|\boldsymbol{Y}-\boldsymbol{Z}_{\theta} \hat{\gamma_{\theta}}\right\|^{2}
$$

其中 $\|\cdot\|$ 表示向量的 $l_{2}$ 范数. 然而, 最小化 $\operatorname{RSS}(\theta)$ 虽然简便, 但是得到的周期估计往往不正确. 众所 周知, 在最小二乘回归中, 回归模型中的解释变量越多, 残差平方和越小, 因此最小化残差平方和往往 会高估周期. 由于真实周期 $\theta_{0}$ 的倍数 $c \theta_{0}$ 也是序列 $m$ 的周期, 而 $c \theta_{0}$ 对应的残差平方和一定小于真 实周期 $\theta_{0}$, 所以最小化残差平方和得到的周期估计通常是真实周期的倍数. 为了避免这个问题, 我们 可以对较大的周期施加一个较大的惩罚项. 这种想法与 AIC 和 BIC 类似. AIC 和 BIC 是在模型拟合 度与模型复杂度之间找到一个平衡, 我们知道

$$
\mathrm{AIC}=2 k-2 \ln (\hat{L}), \quad \mathrm{BIC}=\ln (n) k-2 \ln (\hat{L}),
$$

其中 $k$ 是模型的有效参数个数, $\hat{L}$ 是模型的似然概率. 在最小二乘回归模型中, 常用的是如下形式的 $\mathrm{AIC}$ 和 BIC:

$$
V=2 k+n \ln (\mathrm{RSS}), \quad \mathrm{BIC}=\ln (n) k+n \ln (\mathrm{RSS})
$$

当然也可以考虑文献 [14] 的准则函数, 形式如下:

$$
\tilde{Q}\left(\theta, \lambda_{T}\right)=\operatorname{RSS}(\theta)+\lambda_{T} \theta
$$

其中 $\lambda_{T}$ 是惩罚参数. 文献 [14] 证明了 $\lambda_{T}$ 要比 $\sigma^{2}$ 稍大, 其中 $\sigma^{2}=\mathrm{E}\left[\varepsilon_{t}^{2}\right]$. 但是, 我们采用 B- 样条逼 近未知趋势之后, 必须对惩罚项加以改变. 结合文献 [17] 中半参部分线性模型下的模型选择方法, 本 文采用的惩罚项的形式为 $(\theta+1) N_{T} \lambda_{T}$, 其中 $N_{T}$ 是 B- 样条基函数的个数, $\lambda_{T}$ 是惩罚参数. 为了保证 周期估计的相合性, 我们应该选择如下形式的惩罚参数:

$$
\lambda_{T}=\sigma^{2} \kappa_{T}
$$

其中 $\kappa_{T}$ 是比 $\log T$ 发散速度稍快的序列. 那么本文中最小化的准则函数 $Q$ 具有如下形式:

$$
Q\left(\theta, \lambda_{T}\right)=\operatorname{RSS}(\theta)+(\theta+1) N_{T} \lambda_{T}
$$

如果采用这种形式的准则函数, 则可以通过最小化 $Q\left(\theta, \lambda_{T}\right)$ 得到 $\theta_{0}$ 的估计

$$
\hat{\theta}=\underset{1 \leqslant \theta \leqslant \Theta_{T}}{\arg \min } Q\left(\theta, \lambda_{T}\right)
$$

实际上, 我们可以将 $(\theta+1) N_{T} \lambda_{T}$ 看作 $l_{0}$ 惩罚, 在模型 (2.4) 中, 模型的有效参数是 $\theta+N_{T}$, 在给定 
节点个数和样条阶数的情形下, $N_{T}$ 是一个常数. 注意到, $Q\left(\theta, \lambda_{T}\right)$ 的形式与 $\mathrm{AIC}$ 和 BIC 也具有一定 的相似性, 在第 4 节的模拟研究中, 我们将比较 $Q\left(\theta, \lambda_{T}\right) 、 \mathrm{AIC}$ 和 BIC 三种准则得到的周期估计的结 果. 需要说明, 本文也将周期估计的问题看成模型选择问题, 这与文献 [13] 的想法相似. 文献 [13] 采用 交叉验证 (cross-validation, CV) 的方法估计周期, 并且比较了 AIC、BIC 和 CV 这三种方法在估计周 期方面的相同点和不同点, 其中 AIC 方法得到的 $\theta_{0}$ 的估计与 CV 方法得到的估计是渐近等价的, 两 者得到的估计都不具有相合性. 而 BIC 方法和文献 [14] 的惩罚最小二乘法得到的周期估计都是相合 估计.

\section{3 未知周期序列 $m$ 和未知趋势 $g$ 的估计}

在上面一小节, 我们通过最小化 $Q\left(\theta, \lambda_{T}\right)$ 得到了周期 $\theta_{0}$ 的估计 $\hat{\theta}$. 一旦给出周期的估计, 就可以 很容易得到周期项 $m$ 和未知趋势 $g$ 的估计. 此时模型 (2.4) 变成一个普通的线性模型, 我们对其作最 小二乘回归就可以得到系数 $\gamma$ 的估计 $\hat{\gamma}_{\hat{\theta}}$, 由下式给出:

$$
\hat{\boldsymbol{\gamma}}_{\hat{\theta}}=\left(\begin{array}{c}
\hat{\boldsymbol{\beta}} \\
\hat{\boldsymbol{\alpha}}
\end{array}\right)=\left(\boldsymbol{Z}_{\hat{\theta}}^{\mathrm{T}} \boldsymbol{Z}_{\hat{\theta}}\right)^{-1} \boldsymbol{Z}_{\hat{\theta}}^{\mathrm{T}} \boldsymbol{Y} .
$$

由于 $\beta=\left(\beta_{1}, \ldots, \beta_{\theta_{0}}\right)^{\mathrm{T}}=\left(m(1), \ldots, m\left(\theta_{0}\right)\right)^{\mathrm{T}}$, 因此, 相应的周期序列 $m$ 的估计为 $\hat{\boldsymbol{\beta}}=(\hat{m}(1), \ldots$, $\hat{m}(\hat{\theta}))^{\mathrm{T}}$, 并且 $\hat{m}(s+k \hat{\theta})=\hat{m}(s)$ 对于任意 $s=1, \ldots, \hat{\theta}$ 和 $k$ 都成立.

同时, 我们也可以得到趋势 $g$ 的估计 $\hat{\boldsymbol{g}}=\boldsymbol{B} \hat{\boldsymbol{\alpha}}$, 其中 $\boldsymbol{B}$ 是前面提到的由 B- 样条基函数在所有时 间点处取值组成的矩阵.

在运用 B- 样条方法时, 我们需要决定基函数的个数和节点的位置. 前面已经说明基函数的个数 与内部节点的个数和基函数的阶数有关, 这些都会影响未知函数的估计效果. 一般而言, 基函数的阶 数对未知函数的估计影响较小, 通常选择三阶样条或者四阶样条. 而节点个数的多少对于未知函数的 估计效果影响较大, 所以我们需要一些方法对节点个数进行选取.

我们知道在局部核估计中, 随着窗宽 $h$ 逐渐变小, 估计的回归曲线越来越不光滑, 在极限情形下, 当 $h \rightarrow 0$ 时, 估计的曲线是样本点之间的连线, 这意味着, 随着窗宽 $h$ 的减小, 偏差会减小而方差会增 大; 反之, 随着窗宽的逐渐增大, 估计的回归曲线会越来越光滑, 在极限情形下, 当 $h \rightarrow \infty$ 时, 估计的 回归曲线是一条直线, 所以当窗宽增大时, 偏差会变大而方差会减小. 相应地, 在 B- 样条估计中, 节点 个数越多, $h$ 越小, 拟合值与真实值的偏差越小, 方差越大; 节点个数越少, 拟合值与真实值的偏差越大, 方差越小. 理论上可以通过最小化方差和偏差两者之和来求得最优节点数, 常用的选择节点数的方法 有 GCV (generalized cross-validation)、 KCV (K-fold cross-validation) 和 BIC 等. 在实际操作中, 我们 也可以选择 $k_{c} T^{1 / 5}$ 附近的节点数, 在文献 [18] 中, $k_{c}$ 的默认值是 2 . 我们可以将区间 $\left[0.2 * T^{\frac{1}{5}}, 5 * T^{\frac{1}{5}}\right]$ 等分成若干份, 然后选择使得 GCV 或 BIC 最小的节点数.

一般而言, 节点位置的选择对未知函数的估计效果影响也较小, 但对某些数据影响可能较大, 尤其 是分布不均匀的数据. 通常而言, 放置节点的方法有两种, 一种是等距放置, 也是本文采用的方法; 另 一种是利用样本分位数, 在样本比较多的区间放置更多的节点.

由于周期估计的方法不应该对节点或者基函数的个数变化过于敏感, 同时也为了方便计算, 所以 在未知周期估计中, 我们选择的内部节点个数 $N \sim T^{1 / 5}$. 得到周期估计 $\hat{\theta}$ 之后, 为了得到更为准确的 趋势估计, 我们通过前面提到的节点个数选择法选择内部节点数, 当然通过 GCV $\mathrm{KCV}$ 和 BIC 等方 
法选出的内部节点数一般也在 $N \sim T^{1 / 5}$ 的附近.

\section{$2.4 \sigma^{2}$ 的简单估计}

注意到, 惩罚参数 $\lambda_{T}=\sigma^{2} \kappa_{T}$ 含有一个未知项 $\sigma^{2}$, 因此需要用估计值 $\tilde{\sigma}^{2}$ 代替 $\sigma^{2}$. 前面讨论过最 小化 $\operatorname{RSS}(\theta)$ 来估计周期得到的估计结果通常为真实周期 $\theta_{0}$ 的倍数, 而不是 $\theta_{0}$. 但是, 当估计 $\sigma^{2}$ 时, 我们可以将 $\tilde{\theta}=\arg \min _{1 \leqslant \theta \leqslant \Theta_{T}} \operatorname{RSS}(\theta)$ 作为 $\theta_{0}$ 的初始估计, 这样做可行的原因在于 $c \theta_{0}$ 也是这个周 期序列的一个周期, 并且 Vogt 和 Linton ${ }^{[14]}$ 证明了 $\mathrm{P}\left(\tilde{\theta}=k \theta_{0}\right) \rightarrow 1$. 得到周期的初始估计后, 我们可 以相应地得到周期序列和趋势函数的估计 $\tilde{m}(t)$ 和 $\tilde{g}\left(\frac{t}{T}\right)$, 估计方法与前面提到的相同. 给定 $\tilde{m}(t)$ 和 $\tilde{g}\left(\frac{t}{T}\right)$, 我们能得到 $\sigma^{2}$ 的一个很直接的估计

$$
\tilde{\sigma}^{2}=\frac{1}{T} \sum_{t=1}^{T}{\tilde{\varepsilon_{t}}}^{2}=\frac{1}{T} \sum_{t=1}^{T}\left(Y_{t}-\tilde{m}(t)-\tilde{g}\left(\frac{t}{T}\right)\right)^{2} .
$$

\section{3 渐近性质}

\section{1 基本假设}

为了得到估计的渐近性质, 我们给出如下 4 个假设:

假设 3.1 [14] 误差项 $\left\{\varepsilon_{t, T}\right\}$ 是强混合的, 存在某个正常数 $C$ 和 $a<1$ 使得 $\alpha(k) \leqslant C a^{k}$, 其中 $\alpha(k)$ 是混合系数.

假设 $3.2^{[14]}$ 存在某个常数 $0<C<\infty$ 和 $\delta>0$ 使得 $\mathrm{E}\left[\left|\varepsilon_{t, T}\right|^{4+\delta}\right] \leqslant C$.

假设 $3.3^{[14]}$ 趋势函数 $g$ 在 $[0,1]$ 上 2 阶连续可导, 即 $g \in \mathcal{H}^{2}[0,1]$.

假设 $3.4^{[19]}$ 相邻两个节点的距离 $h \sim T^{-1 / 5}$, 即内部节点数 $N \sim T^{1 / 5}$.

假设 3.1-3.3 来自于文献 [14]. 类似地, 本文也不假设误差项 $\left\{\varepsilon_{t, T}\right\}$ 平稳, 但需要对它的相关结构 作出一定的要求. 注意到在估计未知周期时并未考虑误差项的相关结构, 所以在这里要求误差项短期 相关, 且是强混合的. 需要说明的是, 关于假设 3.1 中的混合系数 $\alpha(k)$ 是指数衰减的假设可以放宽至 多项式衰减, 但需要对惩罚项的阶数作出更严格的假设, 这一点在文献 [14] 中也有说明. 假设 3.3 是 趋势函数的光滑条件, 是为了保证趋势估计的渐近正态性. 假设 3.4 是对 B- 样条节点个数的假设, 这 个假设在 B- 样条中也十分常见.

\section{2 周期估计 $\hat{\boldsymbol{\theta}}$ 的相合性}

下面的定理给出了周期估计 $\hat{\theta}$ 的渐近性质.

定理 3.1 若假设 3.1-3.4 成立, 并且存在某个正常数 $C<\infty$ 和一个较小的数 $\delta>0$ 使得 $\Theta_{T} \leqslant C T^{2 / 5-\delta}$ 成立, 惩罚参数 $\lambda_{T}$ 满足 $(\log T) \Theta_{T}^{3 / 2} \ll \lambda_{T} N_{T} \ll T$, 则 $\hat{\theta} \stackrel{\mathrm{P}}{\longrightarrow} \theta_{0}$, 即 $\hat{\theta}$ 是周期 $\theta_{0}$ 的相 合估计.

注意到在上面的定理中, $\Theta_{T}$ 可以随着 $T$ 的增大以一定的速度增大. 如果 $\Theta_{T}$ 不是一个固定常数, 那么 $\Theta_{T}$ 发散到正无穷的速度越快, 我们对惩罚项 $\lambda_{T}$ 的要求就越高, 但是 $\Theta_{T}$ 发散到正无穷的速度 不能比 $T^{2 / 5}$ 快. 


\section{3 周期序列估计 $\hat{m}$ 及趋势估计 $\hat{g}$ 的渐近性质}

定理 3.1 给出了周期估计 $\hat{\theta}$ 的相合性. 前面提到过, 如果已知模型的周期, 那么我们的模型就简 化成普通的半参数部分线性模型, 而部分线性模型的理论已经很成熟了. 接下来讨论周期未知时模型 中线性部分即周期序列估计 $\hat{m}$ 及非线性部分即趋势估计 $\hat{g}$ 的收敛速度和渐近分布.

定理 3.2 若假设 3.1-3.4 成立, 进一步假设 $V_{t_{0}, T}$ 的极限存在, 即 $V_{t_{0}}=\lim _{T \rightarrow \infty} V_{t_{0}, T}$, 则对每一 个时间 $t, t=1, \ldots, T$, 有

$$
\sqrt{T}(\hat{m}(t)-m(t)) \stackrel{d}{\longrightarrow} N\left(0, V_{t_{0}}\right),
$$

其中为了简化书写, 对于每个时间点 $t$, 记 $t_{0}=t-\theta_{0}\left\lfloor t / \theta_{0}\right\rfloor, K_{t_{0}, T}=1+\left\lfloor\left(T-t_{0}\right) / \theta\right\rfloor$, 定义

$$
V_{t_{0}, T}=\boldsymbol{x}_{t_{0}+(k-1) \theta_{0}}^{\mathrm{T}} \Phi^{-1} \Sigma_{1} \Phi^{-1} \boldsymbol{x}_{t_{0}+(k-1) \theta_{0}}
$$

其中 $\boldsymbol{x}_{t_{0}+(k-1) \theta_{0}}^{\mathrm{T}}$ 是 $\boldsymbol{X}_{\theta_{0}}$ 的行向量, $\Phi=\left(\sigma_{i j}\right)_{\theta_{0} \times \theta_{0}}, 1 \leqslant i, j \leqslant \theta_{0}, \sigma_{i j}=\operatorname{Cov}\left(\boldsymbol{x}_{i}-\tilde{\boldsymbol{x}}_{i}(t), \boldsymbol{x}_{j}-\tilde{\boldsymbol{x}}_{j}(t)\right)$, $\tilde{\boldsymbol{x}}_{i}(t)=\mathrm{E}\left(\boldsymbol{x}_{i} \mid t\right), \Sigma_{1}=\sum_{i, j=1}^{T} \operatorname{Cov}\left(\left(\boldsymbol{x}_{i}-\tilde{\boldsymbol{x}}_{i}(t)\right) \varepsilon_{i},\left(\boldsymbol{x}_{j}-\tilde{\boldsymbol{x}}_{j}(t)\right) \varepsilon_{j}\right) / T$.

需要说明, 当周期未知时, 周期序列的估计 $\hat{m}$ 的渐近分布与周期已知时相同, 并且渐近方差 $V_{t_{0}}$ 也相同, 这意味着周期估计中的误差在周期序列估计 $\hat{m}$ 的渐近分布中可以被忽略, 这一点可以从附 录中定理 3.2 的证明中看到. 并且部分线性模型中的线性部分与普通线性模型的渐近分布的收玫速度 相同, 都是 $\sqrt{T}$, 这也是部分线性模型的特点. 同时, 注意到假设了渐近方差的存在性, 且这一假设在 很多情形下都是成立的. 若 $\left\{\varepsilon_{t, T}\right\}$ 是平稳的, 则长期方差很容易计算, 我们可以用样本自协方差并且 结合周期图或者谱密度来计算长期方差. 也有大量的文献研究如何计算一般情形下的长期方差, 如文 献 $[20,21]$ 利用样本自协方差的核加权. 如果用 $\hat{J}_{T}$ 表示长期方差的估计, 那么 $\hat{J}_{T} \equiv \hat{\gamma}_{0}+2 \sum_{j=1}^{T-1} k\left(\frac{j}{\ell_{T}}\right) \hat{\gamma}_{j}$, 其中 $\hat{\gamma}_{j}$ 是样本自协方差函数, $\ell_{T}$ 是窗宽参数, $k(\cdot)$ 是核权函数. 一般而言核权函数 $k(\cdot)$ 必须是对称 的, 并且 $k(0)=1$. 在计算长期方差时常用的一个核函数是 Bartlett 核, 形式如下:

$$
k\left(\frac{j}{\ell_{T}}\right)= \begin{cases}\left(1-\frac{j}{\ell_{T}}\right), & 0 \leqslant j \leqslant \ell_{T}-1, \\ 0, & j>\ell_{T}-1 .\end{cases}
$$

最后给出趋势函数的 B- 样条估计 $\hat{g}$ 的一致收敛速度和渐近分布.

定理 3.3 若假设 3.1-3.4 成立, 则有

(i)

$$
\sup _{u \in[0,1]}|\hat{g}(u)-g(u)|=O_{p}\left(\zeta_{0}\left(N_{T}\right)\left(\sqrt{\frac{N}{T}}+N^{-2}\right)\right),
$$

其中 $\zeta_{0}\left(N_{T}\right)$ 是满足 $\sup _{u \in[0,1]}\|\boldsymbol{B}(u)\| \leqslant \zeta_{0}\left(N_{T}\right)$ 且 $\left(\zeta_{0}\left(N_{T}\right)^{2} N_{T}\right) / T \rightarrow 0$ 的常数数列;

(ii) 对于每一个 $u \in(0,1)$, 假设 $V_{u}=\lim _{T \rightarrow \infty} V_{u, T}$ 存在, 如果低平滑条件成立, 即内部节点数满 足 $T^{1 / 5} N^{-1} \rightarrow 0$, 则有

$$
\sqrt{\frac{T}{N}}(\hat{g}(u)-g(u)) \stackrel{d}{\longrightarrow} N\left(0, V_{u}\right),
$$

其中 $V_{u, T}=\boldsymbol{B}(u)^{\mathrm{T}} \Psi^{-1} \Sigma_{2} \Psi^{-1} \boldsymbol{B}(u) / N, \Psi=\frac{\boldsymbol{B}^{\mathrm{T}} \boldsymbol{B}}{T}, \Sigma_{2}=\sum_{i, j=1}^{T} \boldsymbol{B}\left(u_{i}\right) \boldsymbol{B}\left(u_{j}\right)^{\mathrm{T}} \mathrm{E}\left(\varepsilon_{i} \varepsilon_{j}\right) / T$.

注意到, 趋势估计 $\hat{g}$ 的渐近分布与周期序列 $m$ 已知时得到的趋势估计 $\tilde{g}$ 相同, 这意味着周期序 
列估计中的误差在趋势估计 $\hat{g}$ 的渐近分布中可以被忽略. 同时, 与周期序列的渐近理论相似, 这里也 假设了渐近方差是存在的, 并且这一假设在很多情形下也都是成立的. 这里渐近方差的计算方法与前 面提到的类似, 当 $\left\{\varepsilon_{t, T}\right\}$ 平稳时, 长期方差比较容易计算, 对于一般情形下长期方差的计算, 也可参见 文献 $[20,21]$.

\section{4 模拟研究}

本节利用 Monte Carlo 模拟去探索本文方法的有限样本性质. 本文考虑两种模拟假设, 为了方便 比较, 第一个模拟的假设与文献 [14] 的模拟假设完全相同; 由于趋势函数的变化会影响周期估计的效 果, 为了体现本文改进方法的优越性, 在第二个模拟中, 我们假设趋势函数变化更大. 在两种不同的模 拟假设下, 比较本文方法与 BIC、AIC 和 V\&L 在周期估计方面的表现.

模拟一的假设即文献 [14] 的模拟假设: 趋势函数是一个二次函数, 即

$$
g_{1}(u)=2 u^{2}, \quad u \in[0,1]
$$

周期序列 $m$ 是一个正弦序列, 真实周期为 $\theta_{0}$,

$$
m(t)=\sin \left(\frac{2 \pi}{\theta_{0}} t+\frac{3 \pi}{2}\right)
$$

误差项 $\varepsilon_{t}$ 来自一阶自回归模型 $\operatorname{AR}(1): \varepsilon_{t}=0.45 \varepsilon_{t-1}+\eta_{t}$, 其中 $\eta_{t}$ 取自 $N\left(0, \sigma_{\eta}^{2}\right)$. 我们将通过改变 $\sigma_{\eta}^{2}$ 的值来改变信噪比, 并且比较 3 种方差 $\operatorname{var}\left(\varepsilon_{t}\right)=\sigma^{2}$ 分别等于 $0.25 、 0.50$ 和 0.75 和不同样本量下的周 期估计. 对于每个样本量 $T$ 和方差 $\sigma^{2}$, 考虑的备选周期范围为 $\theta \in[1, T / 2]$, 独立重复模拟 $N=1,000$ 次. 注意到, 为了保证 $\operatorname{var}\left(\varepsilon_{t}\right)=\sigma^{2}$ 分别等于 $0.25 、 0.50$ 和 0.75 , 需要 $\sigma_{\eta}^{2}$ 分别等于 $0.2 、 0.4$ 和 0.6 . 与 模拟一假设的不同之处在于, 模拟二假设趋势函数变化更大:

$$
g_{2}(u)=10 u^{2}, \quad u \in[0,1]
$$

同时, 模拟中的惩罚参数 $\lambda_{T}=\tilde{\sigma}^{2} \kappa_{T}$, 其中 $\kappa_{T}=\log T, \tilde{\sigma}^{2}$ 是 $\sigma^{2}$ 的估计, 第 2 节已经给出了它的估计 方法.

\section{1 模拟一}

由于第 5 节实证研究的全球年平均气温变化存在 60-70 年的周期, 所以在模拟一中, 假设真实周期 $\theta_{0}=60$, 样本量 $T$ 分别等于 160、250 和 500. 图 3 展示了模拟一假设下的周期序列 $m$ 和趋势函数 $g$.

由于周期 $\theta_{0}$ 估计的准确与否会直接影响周期序列的估计效果, 也会影响时间序列的预测准确性, 因此, 得到一个较为准确的周期估计是至关重要的. 本文的模拟研究会重点比较几种方法的周期估计, 对于趋势 $g$ 的估计, 我们给出简单展示.

图 4 描绘了几种方法的周期估计结果, 每幅图从上到下依次是在某一个具体的样本量 $T$ 和方差 $\sigma^{2}$ 下通过 BIC、AIC、本文方法和 V\&L 得到的周期估计 $\hat{\theta}$ 的频数分布直方图, 其中 BIC 和 AIC 方法 也利用 B- 样条逼近趋势函数, 而 V\&L 直接忽略趋势. 为了更清晰地比较模拟结果, 表 1 展示了不同 方法下周期估计的准确率, 即 1,000 次模拟中周期的估计值 $\hat{\theta}$ 恰好等于真实周期 $\theta_{0}$ 的比率. 
从图 4 可以看出, 绝大部分周期的估计值 $\hat{\theta}$ 聚集在真实周期 $\theta_{0}$ 附近. 可以看到, 在不同样本量和 误差假设下, 通过本文方法得到的周期估计的准确率更高, 聚集在真实周期附近的周期估计值 $\hat{\theta}$ 更多, 这说明本文方法表现最好. 当样本量 $T=160$ 时, 数据中只包含两个完整周期. 尽管此时周期估计准 确率较低, 但是从直方图来看, 仍然有大部分的 $\hat{\theta}$ 聚集在真实周期附近. 同时, 从表 1 也可以看出此时

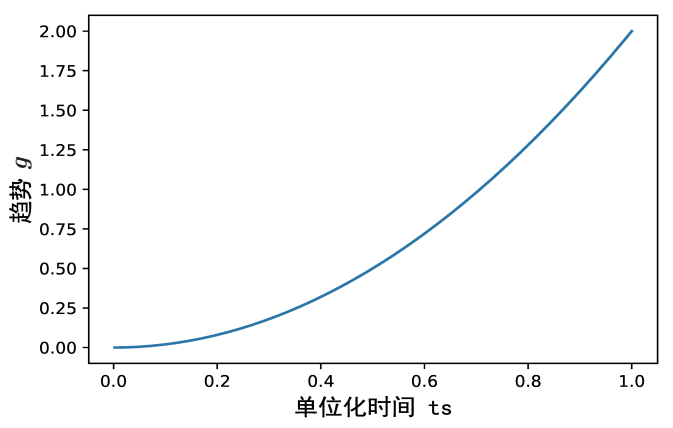

(a)

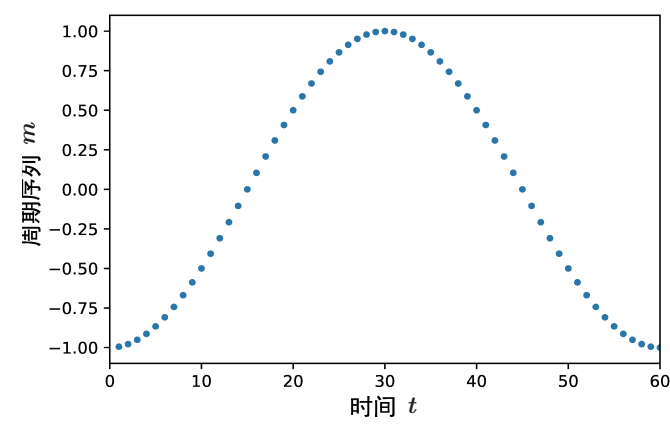

(b)

图 3 (网络版彩图) 趋势函数 $g$ 和周期序列 $m$. (a) 趋势函数 $g ;($ b) 周期序列 $m$

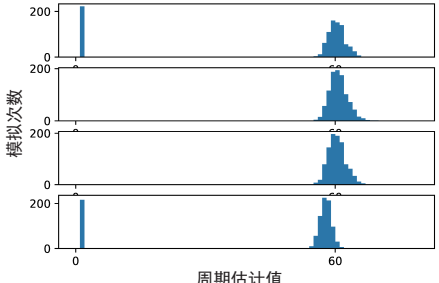

(a)

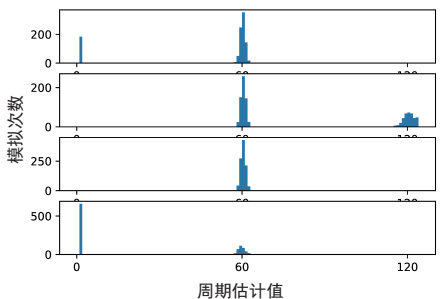

(d)

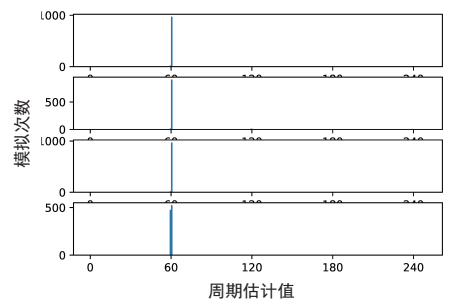

(g)

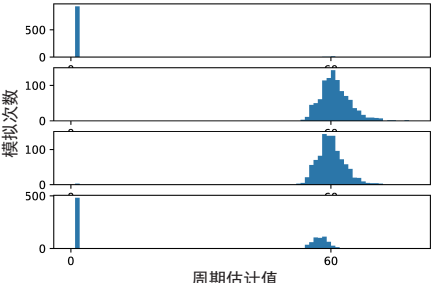

(b)

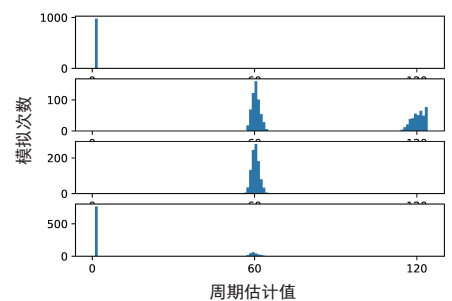

(e)

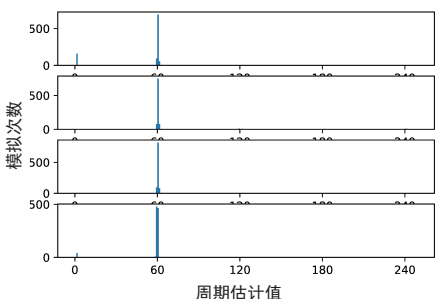

(h)

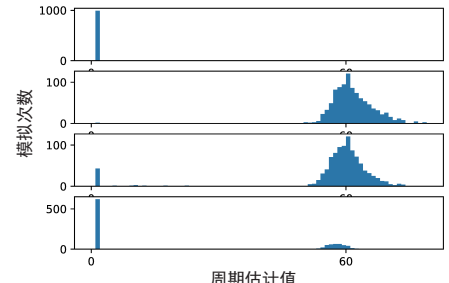

(c)

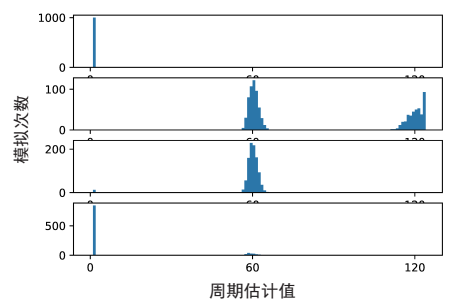

(f)

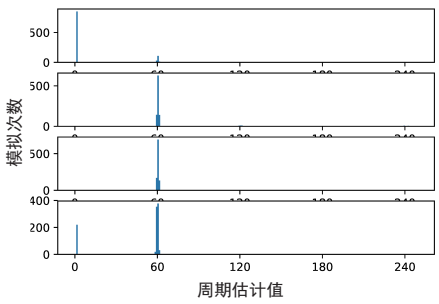

(i)

图 4 (网络版彩图) BIC、AIC、本文方法和 V\&L 在不同样本量 $T$ 和方差 $\sigma^{2}$ 下的周期估计比较. 注意: 每一幅 小图从上到下分别是 BIC、AIC、本文方法和 V\&L. (a) $T=160, \sigma^{2}=0.25 ;$ (b) $T=160, \sigma^{2}=0.50 ;$ (c) $T=160, \sigma^{2}=0.75 ;$ (d) $T=250, \sigma^{2}=0.25 ;$ (e) $T=250, \sigma^{2}=0.50 ;$ (f) $T=250, \sigma^{2}=0.75 ;$ (g) $T=500, \sigma^{2}=0.25 ;(\mathrm{h}) T=500, \sigma^{2}=0.50 ;(\mathrm{i}) T=500, \sigma^{2}=0.75$ 
表 1 BIC、AIC、本文方法和 V\&L 在不同样本量 $T$ 和方差 $\sigma^{2}$ 下的周期估计准确率及均值比较

\begin{tabular}{cccccc}
\hline$T$ & $\sigma^{2}$ & BIC & AIC & Q & V\&L \\
\hline \multirow{2}{*}{160} & 0.25 & $15.2 \%(46.82)$ & $19.4 \%(60.28)$ & $19.3 \%(59.90)$ & $4.5 \%(45.14)$ \\
& 0.50 & $1.3 \%(5.87)$ & $14.5 \%(60.72)$ & $14.9 \%(59.53)$ & $4.1 \%(33.00)$ \\
& 0.75 & $0.2 \%(1.46)$ & $12.1 \%(61.72)$ & $12.1 \%(56.57)$ & $0.4 \%(22.42)$ \\
250 & 0.25 & $35.3 \%(48.96)$ & $25.8 \%(83.73)$ & $42.9 \%(60.00)$ & $8.5 \%(20.95)$ \\
& 0.50 & $0.5 \%(2.34)$ & $16.0 \%(86.50)$ & $28.0 \%(59.84)$ & $4.3 \%(14.63)$ \\
& 0.75 & $0.0 \%(1.11)$ & $12.2 \%(87.30)$ & $22.0 \%(59.34)$ & $2.5 \%(10.08)$ \\
500 & 0.25 & $97.1 \%(59.98)$ & $90.8 \%(68.00)$ & $97.3 \%(59.99)$ & $52.4 \%(59.47)$ \\
& 0.50 & $69.1 \%(50.52)$ & $75.0 \%(71.70)$ & $82.0 \%(59.99)$ & $46.6 \%(57.09)$ \\
& 0.75 & $10.8 \%(9.78)$ & $62.7 \%(70.73)$ & $69.2 \%(59.96)$ & $37.7 \%(46.65)$ \\
\hline
\end{tabular}

$\mathrm{Q}$ 表示本文方法

1,000 次模拟的周期估计均值也基本等于真实周期, 这也说明即使在样本量很少的情形下, 本文方法也 可以得到一个较好的周期估计. 从直方图来看, 随着样本量的增加, 越来越多的 $\hat{\theta}$ 聚集在真实周期附 近, 从表 1 也可以看出, 周期估计的准确率随着样本量的增加逐渐提高并趋近于 1 . 当样本量 $T=500$ 时, 几乎所有的估计值都等于真实周期, 且当误差方差比较小时, 准确率高达 $97.3 \%$.

从直方图 4 可以看出, 随着误差方差 $\sigma^{2}$ 的增大, 周期估计 $\hat{\theta}$ 的分布也越来越分散. 原因在于 随着噪声的增大, 得到一个较为准确的周期估计越来越困难. 当样本量较少且误差的方差较大时, 如 图 4(c), 通过 BIC、本文方法和 V\&L 得到的周期估计有较大一部分等于 1, 但这种现象会随着样本量 的增加而慢慢减少. 然而在样本量相对较多时, 例如, $T=250 、 \sigma^{2}=0.25$, BIC 和 V\&L 周期估计等于 1 的比例仍旧比本文方法高, 这也说明本文方法表现得更好. 实际上, 我们可以通过调节惩罚参数的大 小来降低估计值等于 1 的比例. 估计值等于 1 说明惩罚过大, 由于较小的惩罚参数对周期的惩罚相对 较小, 则会选择较大的周期而不是 1 , 所以可以缩小惩罚参数, 例如, 采用 $0.5 \lambda_{T}$. AIC 方法在样本量较 少时周期估计等于 1 的比例相对较低, 同时, 从表 1 中也可以看到 AIC 在样本量较少时表现较好, 原 因在于 AIC 的惩罚项较小. 但是在样本量相对较多时, AIC 得到的估计有一部分聚集在真实周期的倍 数附近, 这说明此时 AIC 对于周期的惩罚偏小.

为了进一步了解惩罚参数的大小对于周期估计的影响, 图 5 清楚地展示了 $T=500 、 \sigma^{2}=0.5$ 时 准则函数 $Q\left(\theta, \lambda_{T}\right)$ 在不同惩罚参数 $\lambda_{T}$ 下的变化. 从图 5 可以看出, $Q\left(\theta, \lambda_{T}\right)$ 会在真实周期以及它的 倍数附近出现倒尖峰, 如果得到的 $Q\left(\theta, \lambda_{T}\right)$ 图在某些值及它的倍数处出现倒尖峰, 则有理由相信这个 时间序列中存在周期分量. 注意到, 如果惩罚参数较小, 周期估计值会等于真实周期的倍数, 如图 5(a) 所示; 如果惩罚参数较大, 周期估计值会等于 1 , 如图 5(c) 所示. 因此, 选择合适的惩罚参数十分关 键, 否则可能得到错误的结果. 一个合适的惩罚参数应该使得准则函数在第一个倒尖峰处取得全局最 小值, 则在实际情形中, 可以将前面提到的惩罚参数作为一个基准, 在这个基础上进行适当的调节.

图 6 展示了不同样本量和误差下 V\&L 和本文方法得到的趋势估计 $\hat{g}$ 及其 $95 \%$ 置信区间比较, 其 中每一幅小图的左右两幅图分别展示的是 V\&L 和本文方法得到的趋势估计. 从图 6 可以看出, 本文 基于 B- 样条的估计法得到的趋势估计明显比 V\&L 更为准确, 即使在样本量较少的情形下, 例如, 当 $T=160$ 时, 图 6(a) 右图中真实曲线与估计曲线几乎完全重合, 而左边 V\&L 估计的效果相对较差. 同 时, 从上到下, 随着样本量增大, 两种方法得到的趋势估计都越来越接近真实趋势, 置信区间也随着样 本量的增大而变窄. 注意到, 样本量较小时, 趋势估计 $\hat{g}$ 在周期估计 $\hat{\theta}$ 相对准确时也更为准确, 而在周 期估计 $\hat{\theta}$ 相对较差时也相对较差一些, 这也说明了周期估计的准确性会影响趋势估计. 例如, 当样本 量 $T=160 、 \sigma^{2}=0.75$ 时, 周期估计相对较差一些, 此时的趋势估计不如其他情形下准确 (如图 6(c) 所示). 


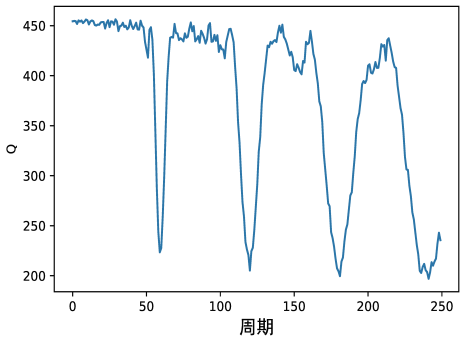

(a)

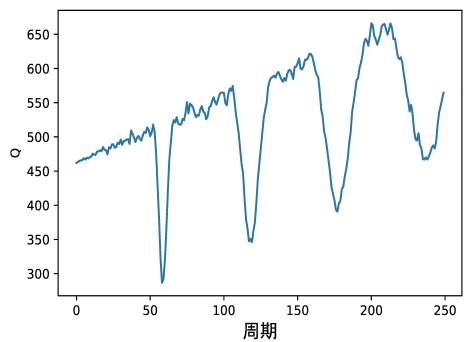

(b)

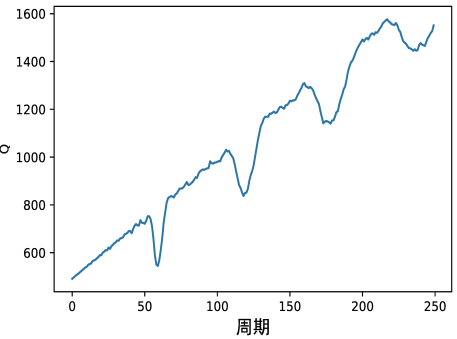

(c)

图 5 (网络版彩图) 当 $T=500 、 \sigma^{2}=0.5$ 时, 准则函数 $Q\left(\theta, \lambda_{T}\right)$ 在不同惩罚参数 $\lambda_{T}$ 下的变化图. (a) $\lambda_{T}^{a}=0.25 \tilde{\sigma}^{2} \log T ;(\mathrm{b}) \lambda_{T}^{b}=\tilde{\sigma}^{2} \log T ;(\mathrm{c}) \lambda_{T}^{c}=4 \tilde{\sigma}^{2} \log T$

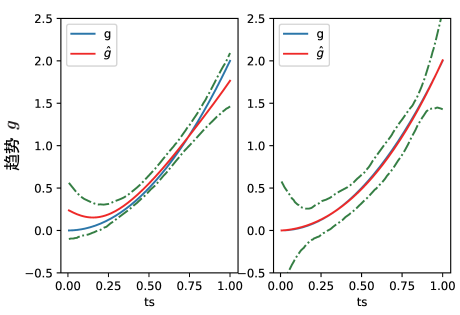

(a)

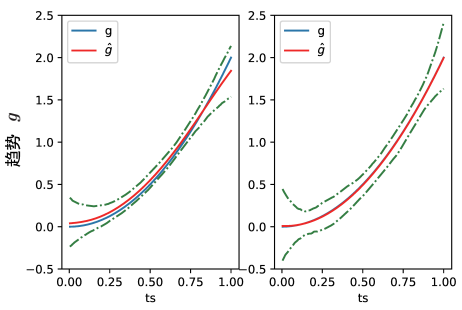

(d)

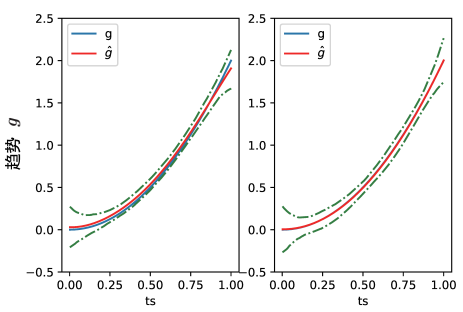

(g)

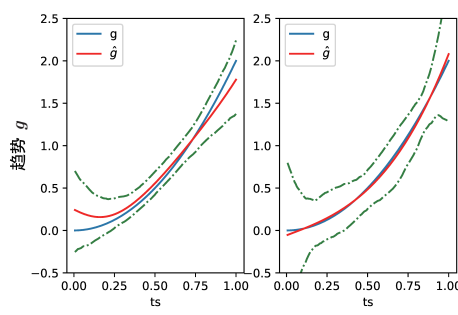

(b)

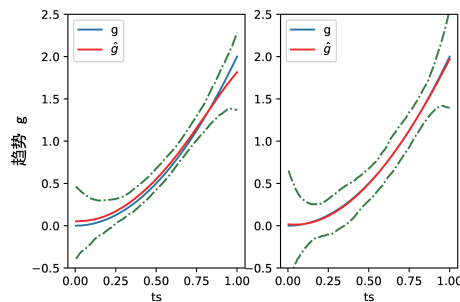

(e)

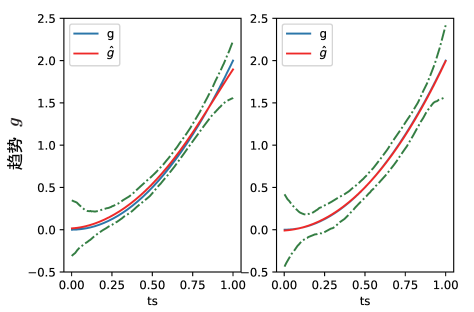

(h)

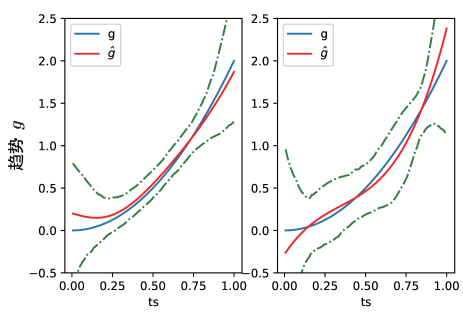

(c)

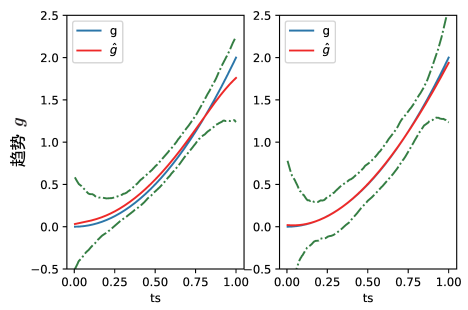

(f)

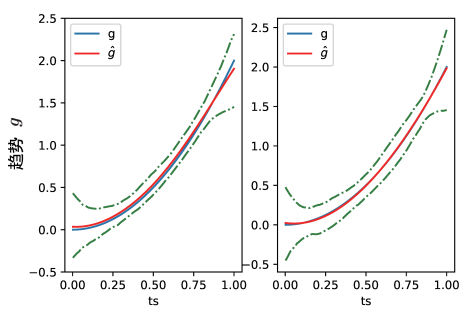

(i)

图 6 (网络版彩图) 本文方法和 V\&L 在不同样本量和误差下的趋势估计结果比较. 注意: 虚线是 $95 \%$ 置信区间, 每一幅小图中左边是基于 $V \& L$ 的趋势估计, 右边是基于本文方法得到的趋势估计. (a) $T=160, \sigma^{2}=0.25$; (b) $T=160, \sigma^{2}=0.50$; (c) $T=160, \sigma^{2}=0.75 ;$ (d) $T=250, \sigma^{2}=0.25$; (e) $T=250, \sigma^{2}=0.50$; (f) $T=250, \sigma^{2}=0.75 ;(\mathrm{g}) T=500, \sigma^{2}=0.25 ;(\mathrm{h}) T=500, \sigma^{2}=0.50 ;$ (i) $T=500, \sigma^{2}=0.75$

\section{2 模拟二}

前面已经说明模拟二假设的趋势函数变化更大: $g_{2}(u)=10 u^{2}, u \in[0,1]$, 周期序列 $m$ 的真实周期 $\theta_{0}$ 为 30 或者 12 .

表 2 展示了不同方法下周期估计的准确率, 其中括号中的值代表每个方法 1,000 次模拟的周期估 
计的平均值. 为了更清楚地比较本文方法与 $\mathrm{V} \& \mathrm{~L}$, 图 7 给出了两种方法周期估计的频数分布直方图. 从表 2 可以看出, 当趋势函数 $g$ 变化较大时, 通过 BIC、AIC 和本文方法这 3 种基于 B- 样条的准则得 到的周期估计准确率明显高于 V\&L 直接忽略趋势项的方法, 周期估计的均值也更接近真实周期 30 .

图 7 表明基于 V\&L 的周期估计几乎都等于 1 或者备选周期上界, 即使我们进一步调节惩罚项也

表 2 当 $\theta_{0}=30$ 时 BIC、AIC、本文方法和 $V \& L$ 在不同样本量 $T$ 和方差 $\sigma^{2}$ 下的周期估计准确率及周期估计 均值比较

\begin{tabular}{cccccc}
\hline$T$ & $\sigma^{2}$ & BIC & AIC & Q & V\&L \\
\hline \multirow{2}{*}{100} & 0.25 & $39.1 \%(26.34)$ & $43.5 \%(30.05)$ & $43.4 \%(29.91)$ & $0.0 \%(47.96)$ \\
& 0.50 & $7.1 \%(7.28)$ & $31.4 \%(30.14)$ & $30.1 \%(29.07)$ & $0.0 \%(43.55)$ \\
& 0.75 & $1.8 \%(2.47)$ & $25.9 \%(30.07)$ & $25.4 \%(28.62)$ & $0.0 \%(40.19)$ \\
\multirow{2}{*}{180} & 0.25 & $94.4 \%(29.99)$ & $53.3 \%(50.71)$ & $94.5 \%(30.00)$ & $0.1 \%(87.36)$ \\
& 0.50 & $55.4 \%(20.88)$ & $42.8 \%(51.01)$ & $77.1 \%(30.02)$ & $0.2 \%(79.57)$ \\
& 0.75 & $14.4 \%(6.67)$ & $33.3 \%(52.90)$ & $64.2 \%(30.16)$ & $0.8 \%(71.44)$ \\
& 0.25 & $99.9 \%(30.00)$ & $78.7 \%(43.80)$ & $99.9 \%(30.00)$ & $0.0 \%(19.00)$ \\
& 0.50 & $93.2 \%(28.58)$ & $67.4 \%(50.75)$ & $97.0 \%(30.09)$ & $0.0 \%(25.76)$ \\
& 0.75 & $45.8 \%(15.00)$ & $60.5 \%(53.45)$ & $91.5 \%(30.12)$ & $0.0 \%(30.82)$ \\
\hline
\end{tabular}

$\mathrm{Q}$ 表示本文方法

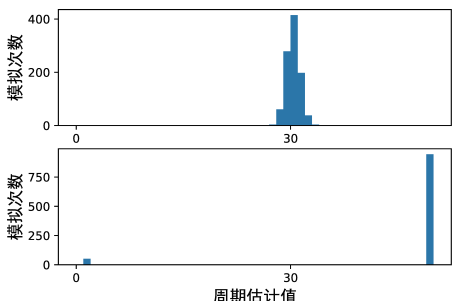

(a)
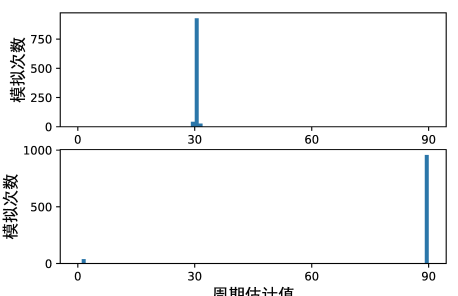

(d)

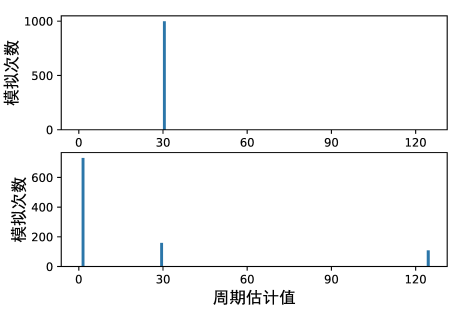

$(\mathrm{g})$

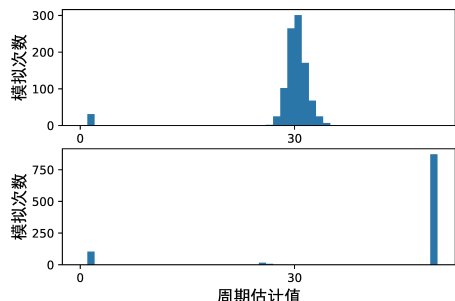

(b)

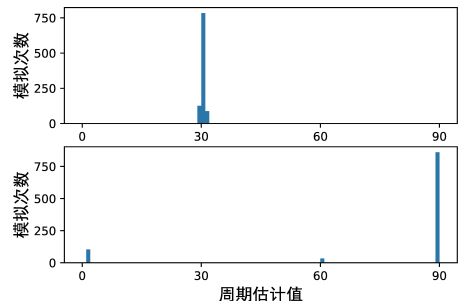

(e)

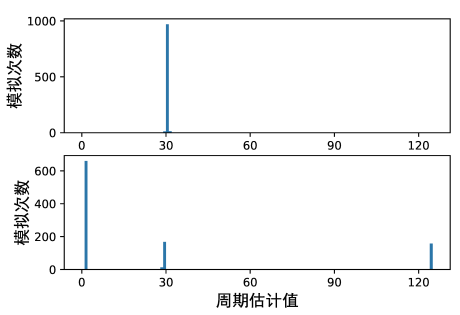

(h)

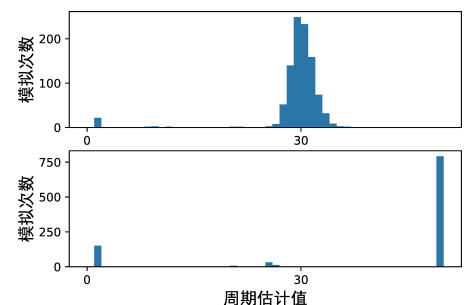

(c)

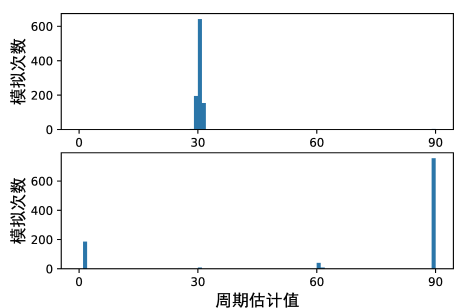

(f)

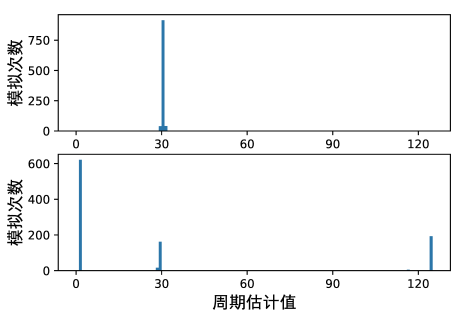

(i)

图 7 (网络版彩图) $\theta_{0}=30$, 本文方法和 $\mathrm{V} \& \mathrm{~L}$ 在不同样本量 $T$ 和方差 $\sigma^{2}$ 下的周期估计比较. 注意: 每一幅 小图从上到下分别是本文方法和 V\&L. (a) $T=100, \sigma^{2}=0.25$; (b) $T=100, \sigma^{2}=0.50 ;$ (c) $T=100$, $\sigma^{2}=0.75$; (d) $T=180, \sigma^{2}=0.25$; (e) $T=180, \sigma^{2}=0.50$; (f) $T=180, \sigma^{2}=0.75$; (g) $T=250$, $\sigma^{2}=0.25$; (h) $T=250, \sigma^{2}=0.50$; (i) $T=250, \sigma^{2}=0.75$ 
难以选出正确的周期. 而本文基于 B- 样条的方法在大部分情形下都能选择出正确的周期, 并且随着 样本量的增加, 聚集在真实周期附近的 $\hat{\theta}$ 越来越多, 周期估计的准确率也逐渐提高并趋近于 1 . 尽管 在样本量较少的情形下, 通过本文方法得到的周期估计准确率相对较低, 但绝大多数周期估计值都在 真实周期附近聚集, 而且 1,000 次估计的平均值也与真实周期十分接近. 例如, 在 $T=100 、 \sigma^{2}=0.25$ 时, 尽管周期估计准确率为 $43.4 \%$, 但平均值为 $29.91 \approx 30$, 这也体现了本文方法具有较好的小样本性 质. 当样本量 $T=250$ 时, 准确率高达 $99.9 \%$, 平均值也几乎等于真实周期, 这也说明了本文方法的相 合性.

表 3 展示了真实周期为 12 时不同方法周期估计的准确率. 考虑真实周期为 12 的原因在于第 5.2 小节中的应用数据是月度数据, 周期为 12 . 表 3 的结果与表 2 相似, 可以看出, 当趋势函数 $g$ 变化较 大时, 通过 BIC、AIC 及本文方法这 3 种基于 B- 样条的准则得到的周期估计准确率明显高于 V\&L, 周期估计的均值也更接近真实周期 12 . 即使在样本量较少的情形下, 周期估计准确率也较高, 例如, 当 $T=50 、 \sigma^{2}=0.25$ 时, 本文方法的周期估计准确率高达 $85.6 \%$. 同时相比较 BIC 和 AIC 而言, 本文方 法对误差方差 $\sigma^{2}$ 的变化没有那么敏感, 例如, 当样本量 $T=70$ 和 $T=100$ 时, 本文方法的周期估计 准确率基本都在 $95 \%$ 以上, 而 BIC 和 AIC 方法在误差方差较大时表现较差. 当样本量较多时, 例如, 当 $T=100$ 时, 基于本文方法的周期估计准确率几乎为 $100 \%$, 模拟结果也展现了本文方法得到的周 期估计是相合的.

图 8 展现了在趋势函数变化较大的情形下基于 V\&L 和本文方法得到的趋势估计 $\hat{g}$ 的结果, 其中 每一幅小图的左右两幅图分别展示的是基于 V\&L 和本文方法得到的趋势估计. 与图 6 相比, 此时两 种估计方法得到的趋势估计效果的差异更加明显, V\&L 已经不能较好地拟合趋势, 而本文基于 B- 样 条的估计法仍然能够十分准确地估计趋势函数. 根本原因在于, 模拟二中趋势函数变化较大, V\&L 的 周期估计准确率很低, 导致其趋势估计效果也较差. 而在模拟一中趋势函数变化较小, 基于 V\&L 得到 的周期估计结果相比模拟二较好, 相应地, 图 6 趋势估计的结果相比图 8 也较好一些. 本文方法在两 个模拟设定中的周期估计表现都比较好, 趋势估计也都十分准确, 这也说明了周期估计的结果会影响 趋势估计的结果, 一个准确的周期估计是十分重要的.

为了进一步展现趋势项对周期估计的影响, 也进一步说明本文方法的优越性, 表 4 比较了本文方 法和 V\&L 在不同趋势函数下的周期估计. 趋势项 $g(u)=K u^{2}-K / 3, u \in[0,1]$, 我们通过改变 $K$ 的值 改变趋势函数的变化幅度, $\sigma^{2}=0.5$, 真实周期 $\theta_{0}=12$, 其他设定与模拟一相同.

从表 4 可以看到, 通过 BIC、AIC 和本文方法得到的周期估计的准确率基本不会随着趋势变化幅 度 $K$ 的变化而变化, 原因在于利用 B- 样条逼近了未知趋势项, 从而使得本文估计方法的效果基本不 受趋势变化幅度的影响. 而通过 V\&L 得到的周期估计的准确率随着 $K$ 增大而降低, 当 $K=10$ 时, 周

表 3 真实周期 $\theta_{0}=12$ 时不同方法在不同样本量 $T$ 和方差 $\sigma^{2}$ 下的周期估计准确率比较

\begin{tabular}{cccccc}
\hline$T$ & $\sigma^{2}$ & BIC & AIC & Q & V\&L \\
\hline 50 & 0.25 & $85.6 \%$ & $35.1 \%$ & $88.5 \%$ & $0.0 \%$ \\
& 0.50 & $58.7 \%$ & $25.9 \%$ & $70.1 \%$ & $0.0 \%$ \\
70 & 0.75 & $36.9 \%$ & $19.8 \%$ & $56.8 \%$ & $0.0 \%$ \\
& 0.25 & $98.8 \%$ & $56.8 \%$ & $98.8 \%$ & $11.0 \%$ \\
\multirow{2}{*}{100} & 0.50 & $85.6 \%$ & $46.0 \%$ & $93.5 \%$ & $16.1 \%$ \\
& 0.75 & $59.0 \%$ & $37.8 \%$ & $76.2 \%$ & $14.5 \%$ \\
& 0.25 & $100.0 \%$ & $51.8 \%$ & $100.0 \%$ & $0.7 \%$ \\
\hline
\end{tabular}

$\mathrm{Q}$ 表示本文方法 


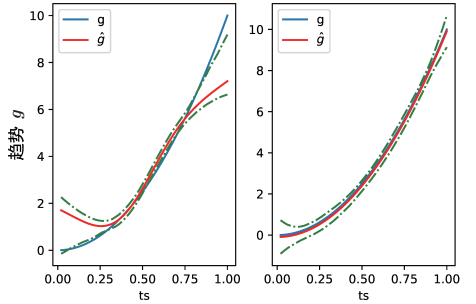

(a)

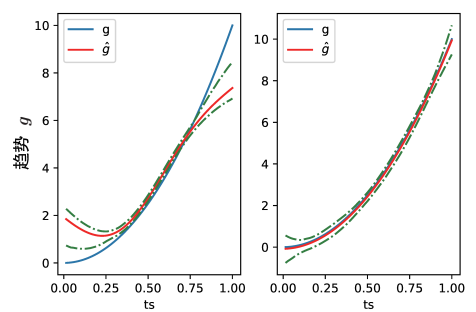

(d)

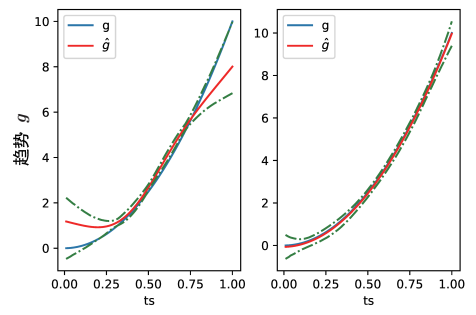

(g)

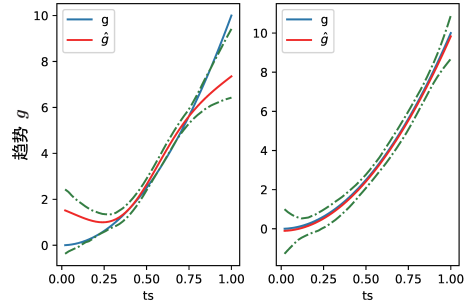

(b)

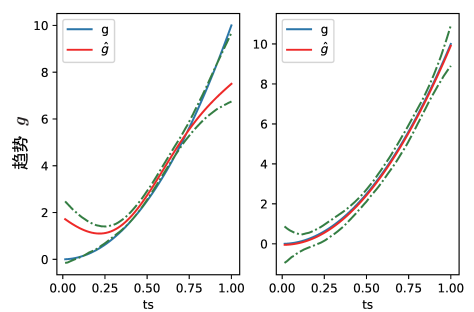

(e)

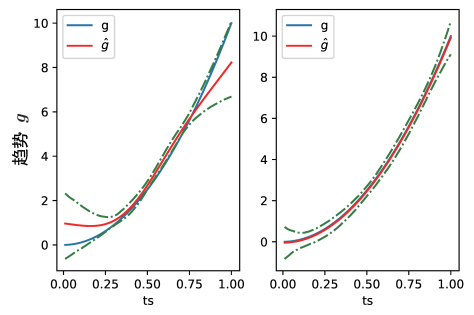

(h)

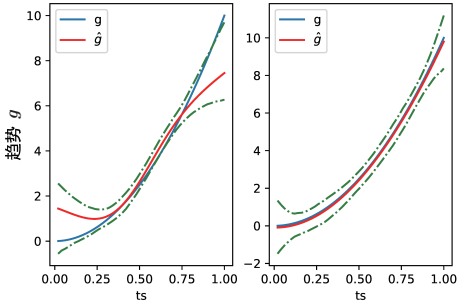

(c)

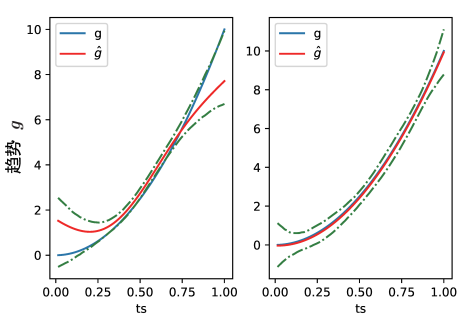

(f)

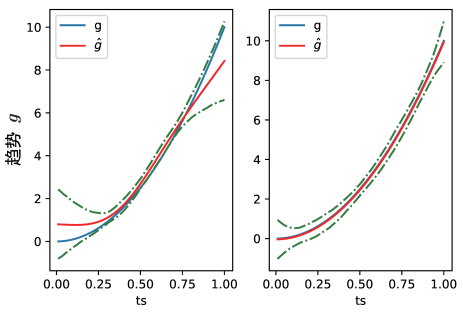

(i)

图 8 (网络版彩图) $\theta_{0}=12$, 本文方法和 $\mathrm{V} \& \mathrm{~L}$ 在不同样本量和误差下的趋势估计结果比较. 注意: 虚线是 $95 \%$ 置 信区间, 每一幅小图中左边是基于 $V \& L$ 的趋势估计, 右边是基于本文方法得到的趋势估计. (a) $T=50, \sigma^{2}=0.25$; (b) $T=50, \sigma^{2}=0.50$; (c) $T=50, \sigma^{2}=0.75$; (d) $T=70, \sigma^{2}=0.25$; (e) $T=70, \sigma^{2}=0.50$; (f) $T=70, \sigma^{2}=0.75 ;(\mathrm{g}) T=100, \sigma^{2}=0.25 ;$ (h) $T=100, \sigma^{2}=0.50 ;$ (i) $T=100, \sigma^{2}=0.75$

表 $4 \sigma^{2}=0.5$ 、真实周期 $\theta_{0}=12$ 时不同方法在不同趋势函数变化幅度 $K$ 下的周期估计准确率比较

\begin{tabular}{rccccrrr}
\hline$T$ & 准则 & $K=0.5$ & $K=2$ & $K=4$ & $K=6$ & $K=8$ & \multicolumn{1}{c}{$K=10$} \\
\hline 50 & BIC & $61.3 \%$ & $62.0 \%$ & $61.0 \%$ & $62.5 \%$ & $61.0 \%$ & $60.3 \%$ \\
& AIC & $26.8 \%$ & $26.2 \%$ & $25.9 \%$ & $28.4 \%$ & $24.2 \%$ & $27.0 \%$ \\
& Q & $74.5 \%$ & $74.2 \%$ & $74.1 \%$ & $74.9 \%$ & $74.1 \%$ & $73.0 \%$ \\
\multirow{5}{*}{100} & V\&L & $71.4 \%$ & $47.4 \%$ & $11.3 \%$ & $0.3 \%$ & $0.0 \%$ & $0.0 \%$ \\
& BIC & $96.9 \%$ & $97.6 \%$ & $98.1 \%$ & $98.4 \%$ & $97.6 \%$ & $97.8 \%$ \\
& AIC & $47.2 \%$ & $47.2 \%$ & $47.3 \%$ & $47.3 \%$ & $42.5 \%$ & $44.3 \%$ \\
& Q & $98.5 \%$ & $99.4 \%$ & $99.3 \%$ & $99.4 \%$ & $99.2 \%$ & $99.5 \%$ \\
& V\&L & $98.5 \%$ & $97.3 \%$ & $72.7 \%$ & $49.0 \%$ & $18.3 \%$ & $1.7 \%$ \\
\hline
\end{tabular}

$\mathrm{Q}$ 表示本文方法

期估计准确率几乎为 0 . 这也说明了当趋势变化较大时, 直接忽略趋势的做法存在一定的缺陷. 图 9 给 出一个更为直观的展示, 随着趋势变化幅度 $K$ 的增大, V\&L 忽略趋势的方法得到的周期估计会越来 越分散, 并且聚集在真实值附近的估计值越来越少, 而通过本文方法得到的估计值的分布则基本不变. 


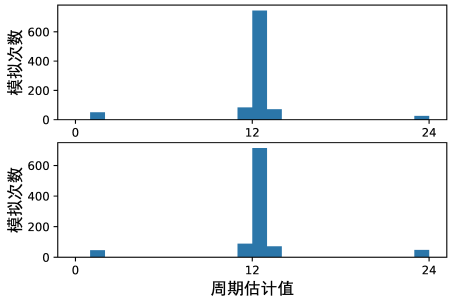

(a)

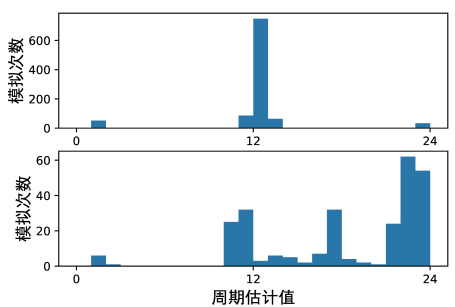

(d)

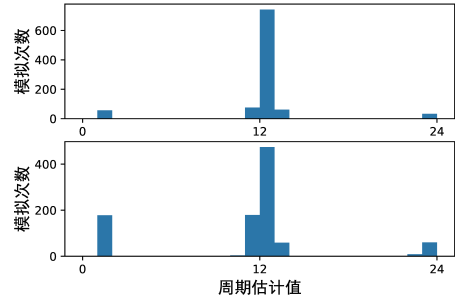

(b)

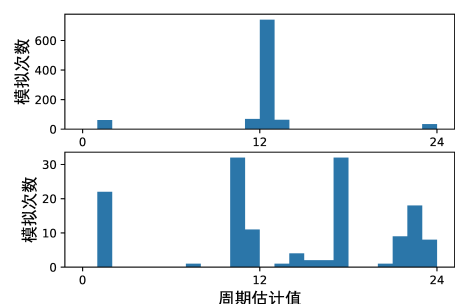

(e)

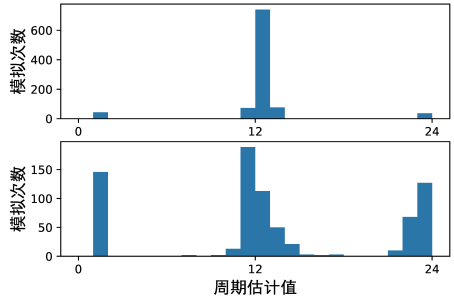

(c)

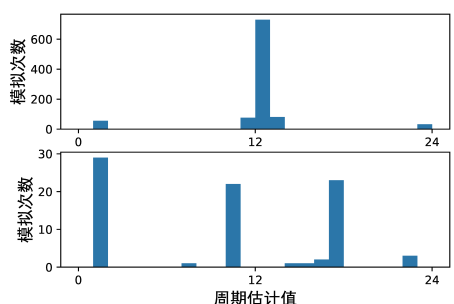

(f)

图 9 (网络版彩图) $T=50, \sigma^{2}=0.5$, 真实周期 $\theta_{0}=12$, 本文方法和 $\mathrm{V} \& \mathrm{~L}$ 在不同趋势函数变化幅度 $K$ 下的 周期估计比较, 注意: 每一幅小图中上图是本文方法, 下图是 $\mathrm{V} \& \mathrm{~L}$. (a) $K=0.5 ;$ (b) $K=2 ;(\mathrm{c}) K=4 ;(\mathrm{d})$ $K=6$; (e) $K=8$; (f) $K=10$

\section{5 实证研究}

本节将基于 B- 样条的惩罚最小二乘估计法应用于两个实际数据. 第一个数据是 1850-2017 年全 球年平均气温的异常变化, 即全球年平均气温与 1961-1990 年平均气温的偏差 ${ }^{1)}$, 这个实例来自于文 献 [14]. 第二个数据是 1995 年 1 月至 2019 年 4 月我国月度进出口贸易与国内贸易数据, 由于这是月 度数据, 因此可以认为这个数据的周期为 12 . 这两个实际数据都包含时间趋势项和周期分量. 由于周 期项的存在会影响趋势估计, 相应地, 趋势项也会影响周期估计, 所以需要精确地分离周期和趋势分量 才能得到周期项和趋势项较为准确的估计, 然后才可以进一步做出更加准确的预测, 帮助我们更好地 解决问题.

\section{1 全球年平均气温异常变化}

众所周知, 在过去的一百多年里, 全球年平均气温显著升高, 全球变暖已经成为一个不可忽视的 问题. 如果我们能够提出一个可靠的估计变暖趋势的方法, 那么将对人们研究全球变暖有巨大的帮助. 全球气温变化是存在周期性的, 周期大约为 60 到 70 年, 这在很多气候学方面的文献中都有说明. 由 于这种周期性的存在必然会对趋势估计产生影响, 而且并不知道它的具体周期, 所以这需要首先给出 一个较为准确的周期估计, 然后才能进一步估计变暖趋势.

本小节将本文方法应用于全球年平均气温变化数据, 图 10(a) 描绘了 1850-2017 年全球年平均气 温的异常变化, 从时序图可以看出, 全球年平均气温呈现出明显的上升趋势.

我们利用基于 B- 样条的惩罚最小二乘估计法估计全球气温变化的周期, 利用年平均气温 $Y_{t, T}$ 拟

1) 这个数据集是 HadCRUT4, 可以从网站 https://crudata.uea.ac.uk/cru/data/temperature/ 下载, 也可以在此网站上看 到有关此数据的具体说明. 


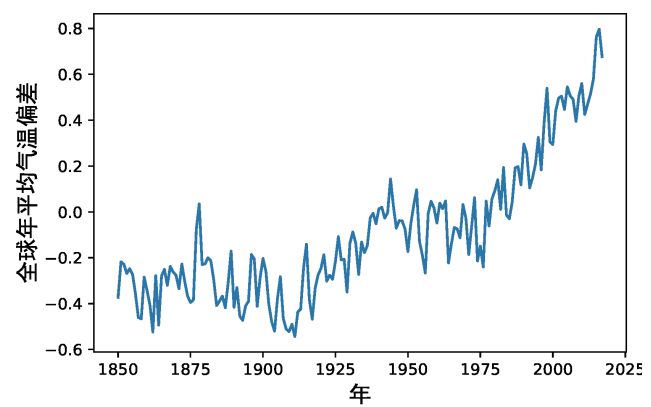

(a)

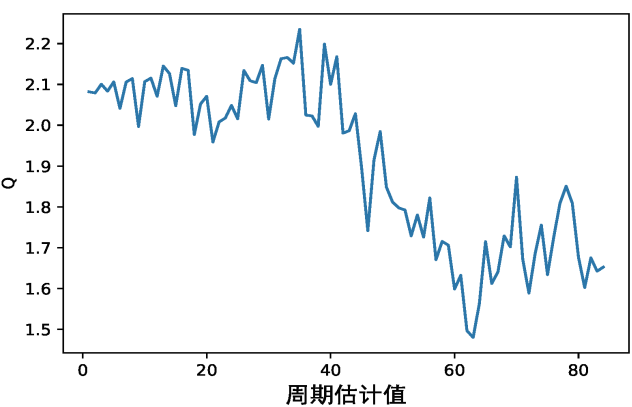

(b)

图 10 (网络版彩图) 1850-2017 年全球年平均气温变化图 (单位: 摄氏度) 及准则函数 $Q\left(\theta, \lambda_{T}\right)$ 变化图. (a) $1850-2017$ 年全球年平均气温变化图; $(b)$ 准则函数 $Q\left(\theta, \lambda_{T}\right)$ 变化图

合模型

$$
Y_{t, T}=g\left(\frac{t}{T}\right)+m(t)+\varepsilon_{t, T},
$$

其中 $T=168, \theta_{0}$ 是待估周期, $m(t)$ 是周期序列, $g$ 是未知趋势函数. 同样地, 首先利用 B- 样条逼近未 知趋势 $g$, 则

$$
\boldsymbol{Y}=\boldsymbol{B} \boldsymbol{\alpha}+\boldsymbol{X}_{\theta_{0}} \boldsymbol{\beta}+\boldsymbol{\varsigma}=\boldsymbol{Z}_{\theta_{0}} \boldsymbol{\gamma}+\boldsymbol{\varsigma},
$$

其中 $\boldsymbol{\varsigma}=\boldsymbol{\varepsilon}+\boldsymbol{g}-\boldsymbol{B} \boldsymbol{\alpha}$. 类似地, 对于 B- 样条基函数个数的选择, 我们采用 4 阶样条, 利用 GCV 去选 择节点个数. 在此应用中, 通过 GCV 选择的内部节点数是 3 个.

然后通过最小化准则函数 $Q\left(\theta, \lambda_{T}\right)=\operatorname{RSS}(\theta)+\lambda_{T} N_{T}(\theta+1)$ 来估计未知周期, 其中惩罚参数 $\lambda_{T}=\tilde{\sigma}^{2} \log T$. 如图 10(b) 所示, 准则函数 $Q\left(\theta, \lambda_{T}\right)$ 在 $\hat{\theta}=63$ 处取得最小值, 且存在明显的倒尖峰现 象, 所以认为全球年气温变化呈现出周期变化, 且周期是 63 .

得到周期估计后, 我们可以通过最小二乘估计很容易得到周期序列的估计 $\hat{m}$ 和趋势函数的估计 $\hat{\boldsymbol{g}}=\boldsymbol{B} \hat{\boldsymbol{\alpha}}$. 图 11(a) 是一个完整周期 63 年的估计图, 图 11(b) 是经过平滑后的周期序列的估计图, 图 11(b) 中浅色曲线代表剔除趋势估计后的全球年平均气温序列, 即 $Y_{T}-\hat{g}\left(\frac{t}{T}\right)$, 可以看到, 周期序列的 拟合效果较好, 全球年平均气温呈现周期变化.

趋势项的估计 $\hat{\boldsymbol{g}}$ 展示在图 11(c) 和 11(d) 中, 其中图 11(c) 中的虚线表示趋势估计的 $95 \%$ 逐点 置信区间, 图 11(d) 中的浅色曲线表示剔除周期序列估计后的全球年平均气温序列, 即 $Y_{T}-\hat{m}(t)$. 从 图 11(c) 和 11(d) 可以看出, 全球年平均气温呈现出二次函数的变化, 年平均气温呈现二次函数变化 的这一特点在很多文献中都有说明.

残差 $\hat{\varepsilon_{t}}=Y_{t}-\hat{m}(t)-\hat{g}\left(\frac{t}{T}\right)$ 的估计图如图 12 所示, 从残差序列图以及残差的自相关和偏自相关 图可以看出, 残差序列不存在明显的趋势性和周期性, 这也说明本文方法可以很好地提取数据的趋势 成分和周期成分.

我们对残差进行进一步建模, 拟合了 $\mathrm{MA}(3)$ 模型 $\varepsilon_{t}=\eta_{t}+0.43 \eta_{t-2}+0.56 \eta_{t-3}$, 并且该模型通过 了模型诊断, 拟合后的剩余残差不再存在相关关系, 这说明 MA(3) 模型可以较好地拟合误差.

\subsection{5 年 1 月至 2019 年 4 月我国月度进出口贸易与国内贸易}

类似地, 我们将本文方法应用于 1995 年 1 月至 2019 年 4 月我国月度进出口贸易与国内贸易的 比较, 其中国内贸易的指标选取的是社会消费品零售总额. 图 13(a) 描绘了 1995 年 1 月至 2019 年 4 
月我国月度进出口总额与社会消费品零售总额的变化, 可以看出, 我国进出口贸易和国内贸易都存在 明显的上升趋势和周期变化.

明显地, 该数据的趋势变化较大, 基于 V\&L 已经不能选择出正确的周期. 但通过本文的方法, 准 则函数 $Q\left(\theta, \lambda_{T}\right)$ 仍在 $\hat{\theta}=12$ 处达到最小, 对于月度数据来说, 周期为 12 会比较合理. 对于社会消费 品零售总额的周期估计, 如图 13(c) 所示, V\&L 的准则函数却在 73 处达到最小值, 即周期估计为 73 , 并且即使调整惩罚参数的系数也无法选择出正确的周期, 这也说明了 V\&L 只适用于趋势变化较小的 数据. 而本文方法仍然能够选出正确周期 (见图 13(b)), 所以我们得出结论: 我国月度进出口总额与社

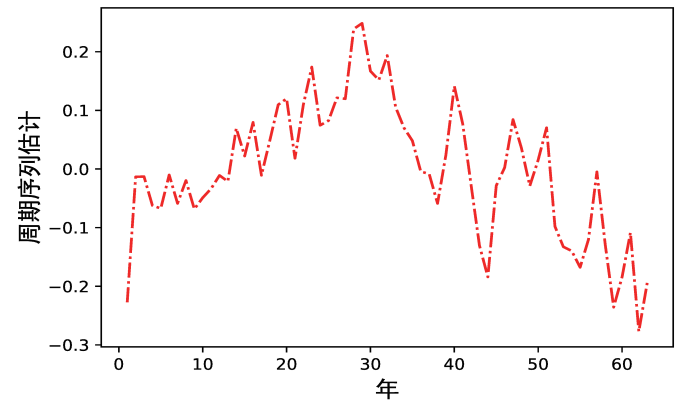

(a)

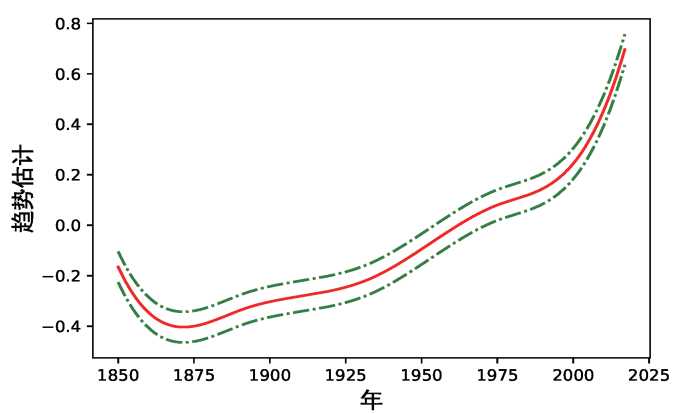

(c)

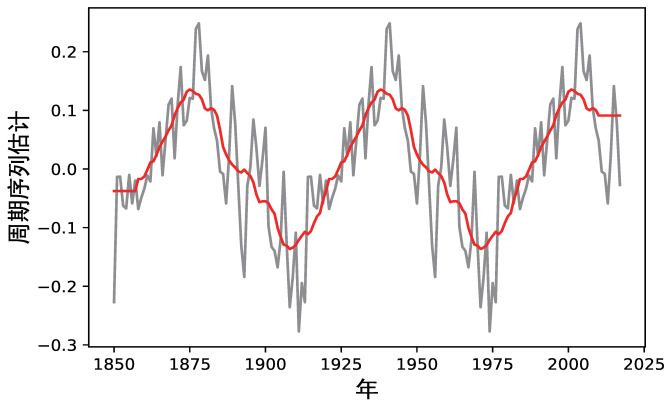

(b)

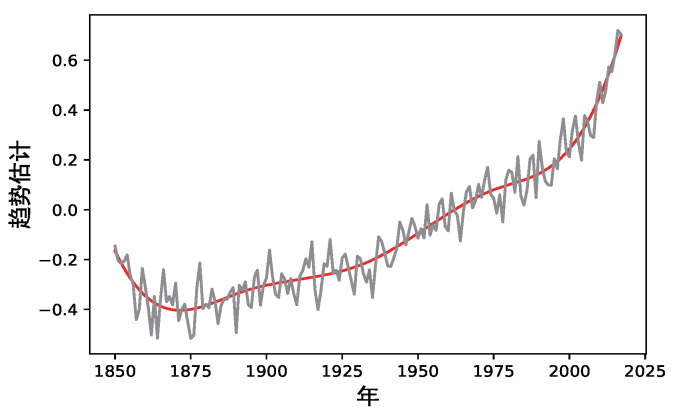

(d)

图 11 (网络版彩图) 周期序列 $m$ 的估计图和趋势 $g$ 的估计图. (a) 一个周期 63 年的序列; (b) 平滑后的整个周 期序列, 浅色曲线表示剔除趋势估计后的序列 $\boldsymbol{Y}_{T}-\hat{g}\left(\frac{t}{T}\right)$; (c) 趋势估计图及其 $95 \%$ 置信区间; (d) 浅色曲表示剔 除周期序列估计后的全球年平均气温序列 $Y_{T}-\hat{m}(t)$

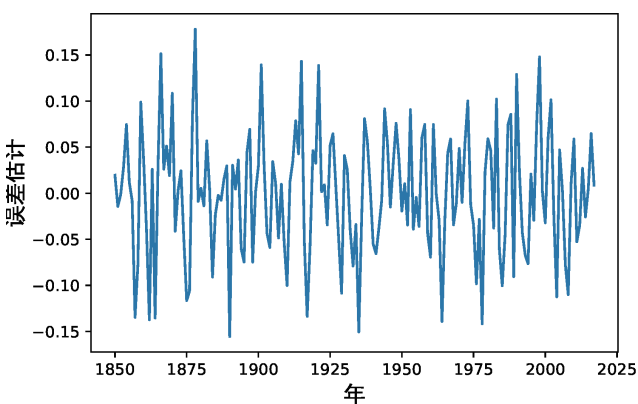

(a)

\begin{tabular}{|c|c|c|c|c|c|c|}
\hline 自相关 & 偏自相关 & & $A C$ & PAC & Q-Stat & Prob \\
\hline 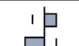 & 12 & 1 & 0.140 & 0.140 & 3.3745 & 0.066 \\
\hline 5 & 득 & & -0.231 & -0.256 & 12.540 & 0.002 \\
\hline 5' & , & & -0.191 & $\begin{array}{r}-0.125 \\
\end{array}$ & 18.883 & 0.000 \\
\hline 111 & יו & 4 & 0.033 & 0.027 & 19.073 & 0.001 \\
\hline 101 & 다 & & -0.062 & -0.159 & 19.740 & 0.001 \\
\hline$C_{1}$ & 14 & & -0.119 & -0.113 & 22.233 & 0.001 \\
\hline יו 1 & $1]$ & & 0.071 & 0.076 & 23.134 & 0.002 \\
\hline 1$]$ & c & 8 & 0.008 & -0.122 & 23.146 & 0.003 \\
\hline d & ב & 9. & -0.129 & -0.140 & 26.117 & 0.002 \\
\hline 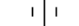 & ו ו 1 & $10-$ & -0.006 & 0.043 & 26.125 & 0.004 \\
\hline ינן & 15 & 11 & 0.048 & -0.080 & 26.550 & 0.0 \\
\hline 111 & 101 & 12. & -0.015 & -0.079 & 26.590 & 0.00 \\
\hline
\end{tabular}

(b)

图 12 (网络版彩图) 残差估计 $\hat{\varepsilon_{t}}$ 以及其自相关和偏自相关图. (a) 残差估计; (b) 自相关和偏自相关图 
会消费品零售总额数据都存在周期变化, 且周期为 12.

同样地, 我们可以通过最小二乘估计很容易得到周期序列的估计 $\hat{m}$ 和趋势函数的估计 $\hat{\boldsymbol{g}}=\boldsymbol{B} \hat{\boldsymbol{\alpha}}$. 图 14(a) 展示的是 1995 年 1 月至 2019 年 4 月月度进出口总额和社会消费品零售总额的趋势估计图, 其中浅色曲线表示剔除周期序列估计后的进出口总额或社会消费品零售总额, 即 $Y_{T}-\hat{m}(t)$. 可以看 到, 进出口总额和社会消费品零售总额均呈现出上升的趋势, 但进出口总额的趋势变化波动更大, 这是 因为进出口受国际形势、国际市场的影响较大. 进出口总额存在三次明显的下降趋势: 2008 年、20142015 年和 2018-2019 年. 2008 年主要是受金融危机的影响; 2014-2015 年下降的原因主要在于外需 低迷, 内需不足, 大宗商品价格下降要素成本上升, 传统优势减弱, 所以我国开始了供给侧结构改革; 2018-2019 年主要是受贸易战的影响.

图 14(b) 展示的是一个周期序列的估计, 可以看到国内贸易变化相比进出口总额的变化滞后一期 或者两期, 并且进出口贸易变化波动比国内贸易大. 9 月、12 月和 1 月进出口总额较高, 我们可以猜 测, 9 月份进出口贸易总额上升的原因可能是受开学季的影响; 受圣诞节的影响, 12 月份的进出口总 额也增加了. 而相对地, 2 月的进出口总额最低, 一个原因在于圣诞节假期结束, 进出口下降; 第二个 重要原因是春节效应, 许多外贸企业会在春节前增加出口, 春节后进出口总额会有明显的下降现象. 而 国内贸易变化波动较小, 12 月和 1 月的社会消费品零售总额较高, 原因主要是春节前居民采购较多, 刺激了国内贸易; 受五一假期和国庆假期的影响, 5 月和 10 月的社会消费品零售总额也有明显的上升 现象.

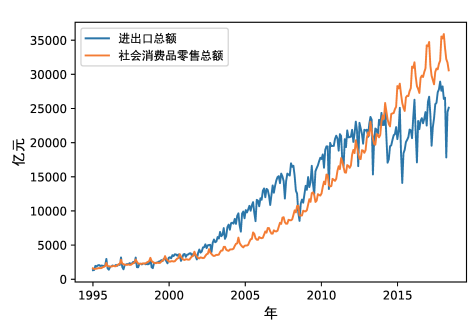

(a)

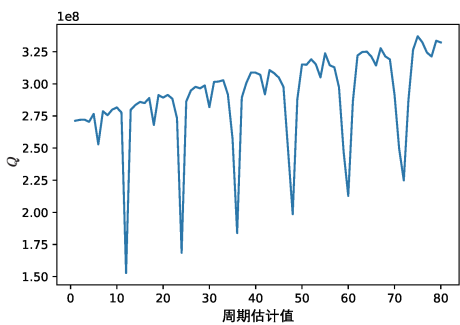

(b)

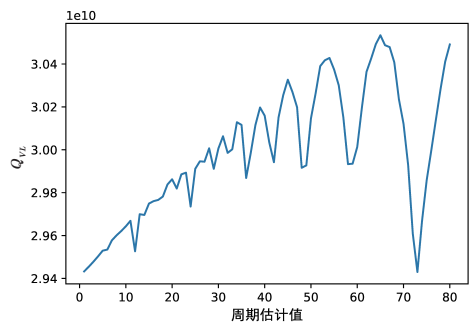

(c)

图 13 (网络版彩图) 1995 年 1 月至 2019 年 4 月月度进出口总额和社会消费品零售总额及准则函数变化图. (a) 进出口总额和社会消费品零售总额; (b) 本文准则函数 $Q ;(\mathrm{c}) \mathrm{V} \& \mathrm{~L}$ 准则函数 $Q_{V L}$

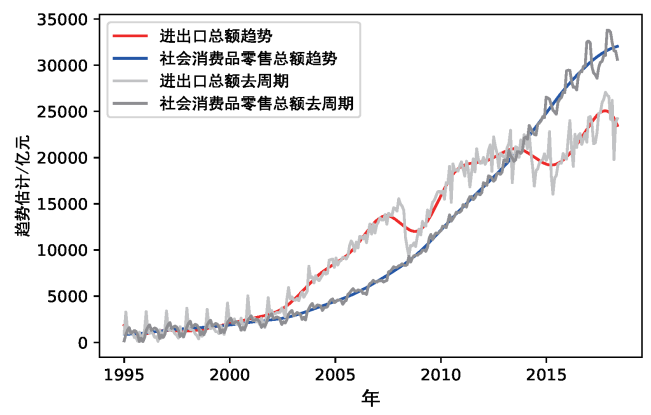

(a)

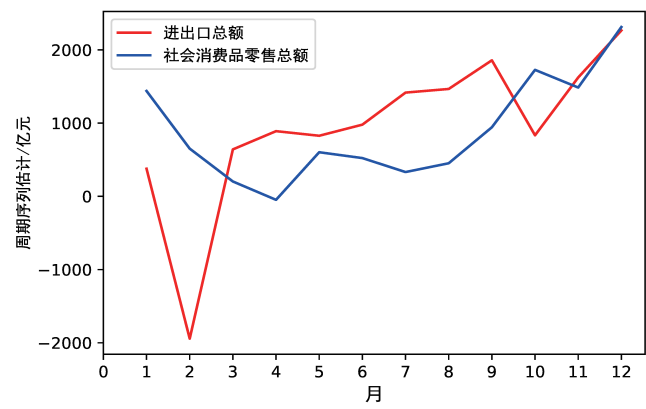

(b)

图 14 (网络版彩图) 1995 年 1 月至 2019 年 4 月月度进出口总额和社会消费品零售总额的趋势估计 $\hat{g}$ 以及周期 估计. (a) 趋势估计 $\hat{g}$, 其中浅色曲线表示剔除周期序列估计后的进出口总额或社会消费品零售总额序列 $\boldsymbol{Y}_{T}-\hat{m}(t)$; (b) 周期序列估计 


\section{6 总结与讨论}

对于含有未知周期和趋势的时间序列, 我们研究了如何对其周期和趋势进行估计, 通过改进 V\&L 来得到更为准确的估计. 本文在估计周期时没有像文献 [14] 一样忽略趋势项, 而是通过 B- 样条逼近 未知时间趋势, 然后运用惩罚最小二乘估计法估计周期、周期序列及趋势. 本文给出了估计量的渐近 性质, 包括周期估计的相合性以及周期序列估计和趋势估计的渐近性质. 我们已经证明通过惩罚最小 二乘法得到的周期估计是相合估计. 同时, 周期估计的误差不会影响周期序列估计和趋势估计的渐近 性质, 并且趋势估计能够达到非参数估计的最优收玫速度. 第 4 节的模拟研究展现了本文方法的优越 性. 与文献 [14] 的方法相比, 基于本文方法的周期估计的准确率更高, 并且更适用于趋势变化较大的 时间序列. 此外, 惩罚最小二乘估计具有较好的小样本性质, 即使在样本量较少时也可以给出较为准 确的周期估计. 最后, 通过全球气温变化数据和我国月度进出口贸易与国内贸易数据的应用说明了本 文方法的实用性.

此外, 根据文献 [14] 可知某些时间序列可能存在多个周期. 假设一个时间序列中含有周期序列 $m_{1}$ 和 $m_{2}$, 相应的周期分别是 $\theta_{1}$ 和 $\theta_{2}$, 则 $m=m_{1}+m_{2}$ 也是周期序列. 如果 $m$ 的最小周期是 $\theta_{0}$, 很多 情形下 $\theta_{0}$ 应该是 $\theta_{1}$ 和 $\theta_{2}$ 的最小公倍数, 当然也有例外, 文献 [22] 给出了具体说明. 如果不考虑序列 的多周期性, 直接运用本文的惩罚最小二乘法就可以得到 $\theta_{0}$ 的估计. 但是, 如果我们还想求解 $m_{1}$ 和 $m_{2}$ 的形式, 需要考虑如下的方法. 令 $\Theta$ 是使得 $m=m_{1}+m_{2}$ 的 $\left(\theta_{1}, \theta_{2}\right)$ 的集合, 一般而言, $\Theta$ 包含很 多对 $\left(\theta_{1}, \theta_{2}\right)$. 一个可行的办法是采用二维的惩罚最小二乘方法, 建立有两个周期分量的模型, 计算其 相应的残差平方和, 然后通过最小化残差平方和与惩罚项的总和得到周期估计, 这也意味着本文的方 法也适用于多周期的序列. 当然在实际应用中可能会需要进一步考虑其他细节, 并且多维情形下的理 论证明也较为复杂, 因为此时设计矩阵 $\boldsymbol{X}_{\theta}$ 的形式变得更为复杂.

在后续的研究中, 我们可以尝试对模型做出改进, 例如, 考虑 M- 估计、检验函数损失和弱化误差 的假设条件等. 注意到, 本文并未将协变量纳入模型, 但在很多情形下, 时间序列可能还受到某些协变 量的影响, 因此可以进一步考虑将协变量纳入模型. 尽管本文模型的误差可以是相关的, 但本文在进 行周期估计时并未考虑误差的具体结构. 所以接下来可以将误差结构纳入估计过程来优化本文的模 型, 以期望得到更加精确的估计, 并使得该方法应用范围更加广泛.

致谢 本文承蒙主编、副主编以及审稿人的审阅指导, 在此感谢他们对本文提出的宝贵意见.

\section{参考文献}

1 Truong Y K. Nonparametric curve estimation with time series errors. J Statist Plann Inference, 1991, 28: 167-183

2 Shao Q, Yang L J. Autoregressive coefficient estimation in nonparametric analysis. J Time Series Anal, 2011, 32: $587-597$

3 Shao Q, Yang L. Oracally efficient estimation and consistent model selection for auto-regressive moving average time series with trend. J R Stat Soc Ser B Stat Methodol, 2017, 79: 507-524

4 Xue L, Yang L. Additive coefficient modeling via polynomial spline. Statist Sinica, 2006, 16: 1423-1446

5 Walker A M. On the estimation of a harmonic component in a time series with stationary independent residuals. Biometrika, 1971, 58: 21-36

6 Hannan E J. The estimation of frequency. J Appl Probab, 1973, 10: 510-519

7 Rice J A, Rosenblatt M. On frequency estimation. Biometrika, 1988, 75: 477-484

8 Quinn B G, Thomson P J. Estimating the frequency of a periodic function. Biometrika, 1991, 78: 65-74

9 Hall P, Reimann J, Rice J. Nonparametric estimation of a periodic function. Biometrika, 2000, 87: 545-557

10 Hall P, Yin J. Nonparametric methods for deconvolving multiperiodic functions. J R Stat Soc Ser B Stat Methodol, 2003, 65: 869-886 
11 Genton M G, Hall P. Statistical inference for evolving periodic functions. J R Stat Soc Ser B Stat Methodol, 2007, 69: 643-657

12 Hall P, Li M. Using the periodogram to estimate period in nonparametric regression. Biometrika, 2006, 93: 411-424

13 Sun Y, Hart J D, Genton M G. Nonparametric inference for periodic sequences. Technometrics, 2012, 54: 83-96

14 Vogt M, Linton O. Nonparametric estimation of a periodic sequence in the presence of a smooth trend. Biometrika, 2014, 101: 121-140

15 Dahlhaus R. Locally stationary processes. Handbook of Statist, 2012, 30: 351-413

16 De Boor C. A Practical Guide to Splines. New York: Springer-Verlag, 1978

17 Bunea F. Consistent covariate selection and post model selection inference in semiparametric regression. Ann Statist, 2004, 32: 898-927

18 Huang J Z, Yang L. Identification of non-linear additive autoregressive models. J R Stat Soc Ser B Stat Methodol, 2004, 66: 463-477

19 Chen H. Convergence rates for parametric components in a partly linear model. Ann Statist, 1988, 16: 136-146

20 Newey W K, West K D. A simple, positive semi-definite, heteroskedasticity and autocorrelation consistent covariance matrix. Econometrica, 1987, 55: 703-708

21 Jong R M, Davidson J. Consistency of kernel estimators of heteroscedastic and autocorrelated covariance matrices. Econometrica, 2000, 68: 407-423

22 Restrepo A, Chacón L P. On the period of sums of discrete periodic signals. IEEE Signal Process Lett, 1998, 5: $164-166$

23 Stone C J. Optimal rates of convergence for nonparametric estimators. Ann Statist, 1980, 8: 1348-1360

24 Stone C J. Optimal global rates of convergence for nonparametric regression. Ann Statist, 1982, 10: 1040-1053

25 Li Q, Racine J S. Nonparametric Econometrics: Theory and Practice. Princeton: Princeton University Press, 2007

\section{附录 A 定理证明}

\section{附录 A.1 定理 3.1 的证明}

为了方便书写, 我们给出一些符号说明. 前面已经给出过, $\boldsymbol{Z}_{\theta_{0}}=\left(\boldsymbol{X}_{\theta_{0}}, \boldsymbol{B}\right), \boldsymbol{Z}_{\theta}=\left(\boldsymbol{X}_{\theta}, \boldsymbol{B}\right)$. 记

$$
\Pi_{\theta}=\boldsymbol{X}_{\theta}\left(\boldsymbol{X}_{\theta}^{\mathrm{T}} \boldsymbol{X}_{\theta}\right)^{-1} \boldsymbol{X}_{\theta}^{\mathrm{T}}
$$

是子空间 $\mathcal{C}\left(\boldsymbol{X}_{\theta}\right)=\left\{X_{\theta} b: b \in \mathbb{R}^{\theta}\right\}$ 上的投影矩阵. 记 $\Delta_{\theta}$ 是空间 $\mathcal{C}\left(\boldsymbol{Z}_{\theta}\right)=\left\{\boldsymbol{Z}_{\theta} d: d \in \mathbb{R}^{\theta+N_{T}}\right\}$ 上的投影 矩阵,

$$
\begin{aligned}
\Delta_{\theta}= & \boldsymbol{Z}_{\theta}\left(\boldsymbol{Z}_{\theta}^{\mathrm{T}} \boldsymbol{Z}_{\theta}\right)^{-1} \boldsymbol{Z}_{\theta}^{\mathrm{T}} \\
= & \left(\boldsymbol{X}_{\theta}, \boldsymbol{B}\right)\left(\left(\begin{array}{c}
\boldsymbol{X}_{\theta}^{\mathrm{T}} \\
\boldsymbol{B}^{\mathrm{T}}
\end{array}\right)\left(X_{\theta}, \boldsymbol{B}\right)\right)^{-1}\left(\begin{array}{c}
\boldsymbol{X}_{\theta}^{\mathrm{T}} \\
\boldsymbol{B}^{\mathrm{T}}
\end{array}\right) \\
= & \left(\boldsymbol{X}_{\theta}, \boldsymbol{B}\right)\left(\begin{array}{c}
\boldsymbol{X}_{\theta}^{\mathrm{T}} \boldsymbol{X}_{\theta} \boldsymbol{X}_{\theta}^{\mathrm{T}} \boldsymbol{B} \\
\boldsymbol{B}^{\mathrm{T}} \boldsymbol{X}_{\theta} \boldsymbol{B}^{\mathrm{T}} \boldsymbol{B}
\end{array}\right) \quad\left(\begin{array}{c}
\boldsymbol{X}_{\theta}^{\mathrm{T}} \\
\boldsymbol{B}^{\mathrm{T}}
\end{array}\right) \\
= & \Pi_{\theta}+\Pi_{\theta} \boldsymbol{B}\left(\boldsymbol{B}^{\mathrm{T}}\left(\boldsymbol{I}-\Pi_{\theta}\right) \boldsymbol{B}\right)^{-1} \boldsymbol{B}^{\mathrm{T}} \Pi_{\theta}-\Pi_{\theta} \boldsymbol{B}\left(\boldsymbol{B}^{\mathrm{T}}\left(\boldsymbol{I}-\Pi_{\theta}\right) \boldsymbol{B}\right)^{-1} \boldsymbol{B}^{\mathrm{T}} \\
& -\boldsymbol{B}\left(\boldsymbol{B}^{\mathrm{T}}\left(\boldsymbol{I}-\Pi_{\theta}\right) \boldsymbol{B}\right)^{-1} \boldsymbol{B}^{\mathrm{T}} \Pi_{\theta}+\boldsymbol{B}\left(\boldsymbol{B}^{\mathrm{T}}\left(\boldsymbol{I}-\Pi_{\theta}\right) \boldsymbol{B}\right)^{-1} \boldsymbol{B}^{\mathrm{T}} \\
= & \Pi_{\theta}+\Pi_{\theta} \boldsymbol{C}_{\theta} \Pi_{\theta}-\Pi_{\theta} \boldsymbol{C}_{\theta}-\boldsymbol{C}_{\theta} \Pi_{\theta}+\boldsymbol{C}_{\theta} \\
= & \Pi_{\theta}+\left(\boldsymbol{I}-\Pi_{\theta}\right) \boldsymbol{C}_{\theta}\left(\boldsymbol{I}-\Pi_{\theta}\right) \\
= & \boldsymbol{P}_{\boldsymbol{X}_{\theta}}+\boldsymbol{P}_{\boldsymbol{M}_{\boldsymbol{X}_{\theta}} \boldsymbol{B}},
\end{aligned}
$$


其中上述等式运用了分块矩阵的求逆公式展开, $\boldsymbol{C}_{\theta}=\boldsymbol{B}\left(\boldsymbol{B}^{\mathrm{T}}\left(\boldsymbol{I}-\Pi_{\theta}\right) \boldsymbol{B}\right)^{-1} \boldsymbol{B}^{\mathrm{T}}, \boldsymbol{P}_{\boldsymbol{X}_{\theta}}$ 表示 $\boldsymbol{Z}_{\theta}=\left(\boldsymbol{X}_{\theta}, \boldsymbol{B}\right)$ 在空间 $\mathcal{C}\left(\boldsymbol{X}_{\theta}\right)$ 的投影矩阵, $\boldsymbol{P}_{\boldsymbol{M}_{\boldsymbol{X}_{\theta}} \boldsymbol{B}}$ 表示 $\boldsymbol{Z}_{\theta}=\left(\boldsymbol{X}_{\theta}, \boldsymbol{B}\right)$ 在空间 $\mathcal{C}\left(\left(\boldsymbol{I}-\Pi_{\theta}\right) \boldsymbol{B}\right)$ 即空间 $\mathcal{C}\left(\boldsymbol{M}_{\boldsymbol{X}_{\theta}} \boldsymbol{B}\right)$ 上的 投影矩阵. 那么,

$$
\boldsymbol{I}-\Delta_{\theta}=\boldsymbol{I}-\Pi_{\theta}-\left(\boldsymbol{I}-\Pi_{\theta}\right) \boldsymbol{C}_{\theta}\left(\boldsymbol{I}-\Pi_{\theta}\right) .
$$

因为 $\boldsymbol{X}_{\theta}$ 是正交的, 所以投影矩阵 $\Pi_{\theta}$ 具有一个很简单的形式. 注意到

$$
\boldsymbol{X}_{\theta}^{\mathrm{T}} \boldsymbol{X}_{\theta}=\left(\boldsymbol{I}_{\theta}, \boldsymbol{I}_{\theta}, \ldots\right)\left(\begin{array}{c}
\boldsymbol{I}_{\theta} \\
\boldsymbol{I}_{\theta} \\
\vdots
\end{array}\right)=\left(\begin{array}{ccc}
K_{1, T}^{[\theta]} & & 0 \\
& \ddots & \\
& & \\
0 & & K_{\theta, T}^{[\theta]}
\end{array}\right)
$$

其中 $K_{s, T}^{[\theta]}=1+\lfloor(T-s) / \theta\rfloor(s=1, \ldots, \theta), K_{s, T}^{[\theta]}$ 是样本中满足 $t=s+(k-1) \theta$ 的时间点 $t$ 的个数. 所 以 $K_{s, T}^{[\theta]}$ 的值等于 $\lfloor T / \theta\rfloor$ 或者 $\lfloor T / \theta\rfloor+1$, 即 $K_{s, T}^{[\theta]}=O(T / \theta)$. 记 $D_{\theta}=\left(\boldsymbol{X}_{\theta}^{\mathrm{T}} \boldsymbol{X}_{\theta}\right)^{-1}$, 则

$$
D_{\theta}=\left(\begin{array}{ccc}
1 / K_{1, T}^{[\theta]} & & 0 \\
& \ddots & \\
& & 1 / K_{\theta, T}^{[\theta]}
\end{array}\right)
$$

那么投影矩阵 $\Pi_{\theta}$ 化为如下简单形式:

$$
\Pi_{\theta}=\boldsymbol{X}_{\theta} D_{\theta} \boldsymbol{X}_{\theta}^{\mathrm{T}}=\left(\begin{array}{ccc}
D_{\theta} & D_{\theta} & \cdots \\
D_{\theta} & \ddots \\
\vdots &
\end{array}\right),
$$

相应的残差平方和写成如下形式:

$$
\begin{aligned}
\operatorname{RSS}(\theta) & =\left(\boldsymbol{Y}-\boldsymbol{Z}_{\theta} \hat{\gamma}_{\theta}\right)^{\mathrm{T}}\left(\boldsymbol{Y}-\boldsymbol{Z}_{\theta} \hat{\gamma}_{\theta}\right) \\
& =\left(\left(\boldsymbol{I}-\Delta_{\theta}\right) \boldsymbol{Y}\right)^{\mathrm{T}}\left(\left(\boldsymbol{I}-\Delta_{\theta}\right) Y\right) \\
& =\boldsymbol{Y}^{\mathrm{T}}\left(\boldsymbol{I}-\Delta_{\theta}\right) \boldsymbol{Y} \\
& =\boldsymbol{Y}^{\mathrm{T}}\left(\boldsymbol{I}-\Pi_{\theta}-\left(\boldsymbol{I}-\Pi_{\theta}\right) \boldsymbol{C}_{\theta}\left(\boldsymbol{I}-\Pi_{\theta}\right)\right) \boldsymbol{Y} .
\end{aligned}
$$

引理 $\mathbf{A . 1}$ 定义 $R_{T}^{i}=\boldsymbol{B}(u)^{\mathrm{T}} \boldsymbol{\alpha}-g(u), r_{T}$ 是 B- 样条逼近误差,

$$
r_{T}=\sup _{u \in[0,1]}\left|\boldsymbol{B}(u)^{\mathrm{T}} \boldsymbol{\alpha}-g(u)\right|
$$

则 $r_{T}=O\left(N_{T}^{-2}\right)$.

引理 A.1 证明可参见文献 [16].

最后, 需要特别说明的是符号 $C$ 用于表示一般常数, 在不同的地方可能有不同的值, 并且 $C$ 不依 赖于任何模型参数, 也不依赖于备选周期 $\theta$ 和样本大小 $T$. 
定义好符号之后, 我们开始证明定理 3.1. 我们需要证明 $\mathrm{P}\left(\hat{\theta} \neq \theta_{0}\right)=o(1)$, 那么 $\hat{\theta}=\theta_{0}+o_{p}(1)$, 这 样便完成了证明. 已知

$$
\mathrm{P}\left(\hat{\theta} \neq \theta_{0}\right) \leqslant \sum_{1 \leqslant \theta \leqslant \Theta_{T}, \theta \neq \theta_{0}} \mathrm{P}\left(Q\left(\theta, \lambda_{T}\right) \leqslant Q\left(\theta_{0}, \lambda_{T}\right)\right)
$$

则只需要证明当样本量 $T$ 足够大时, 对于任意 $\theta\left(1 \leqslant \theta \leqslant \Theta_{T}\right)$ 有

$$
\mathrm{P}\left(Q\left(\theta, \lambda_{T}\right) \leqslant Q\left(\theta_{0}, \lambda_{T}\right)\right) \leqslant C\left(\kappa_{T} \Theta_{T}\right)^{-1},
$$

其中 $\left\{\kappa_{T}\right\}$ 是一个缓慢发散到正无穷的序列, 例如 $\kappa_{T}=\log \log T$, 从而周期估计的相合性得以证明. 接 下来证明 (A.1). 对于每一个固定的 $\theta\left(\theta \neq \theta_{0}\right.$ 且 $\left.1 \leqslant \theta \leqslant \Theta_{T}\right)$, 有

$$
\begin{aligned}
\mathrm{P}\left(Q\left(\theta, \lambda_{T}\right) \leqslant Q\left(\theta_{0}, \lambda_{T}\right)\right) & =\mathrm{P}\left(\boldsymbol{Y}^{\mathrm{T}}\left(\boldsymbol{I}-\Delta_{\theta}\right) \boldsymbol{Y} \leqslant \boldsymbol{Y}^{\mathrm{T}}\left(\boldsymbol{I}-\Delta_{\theta_{0}}\right) \boldsymbol{Y}+N_{T} \lambda_{T}\left(\theta_{0}-\theta\right)\right) \\
& =\mathrm{P}\left(V_{\theta} \leqslant-A_{\theta}-2 S_{\theta}^{\boldsymbol{\varepsilon}}-2 S_{\theta}^{\boldsymbol{g}}+2 G_{\theta}^{\varepsilon}+G_{\theta}^{\boldsymbol{g}}+\lambda_{T} N_{T}\left(\theta_{0}-\theta\right)\right),
\end{aligned}
$$

其中

$$
\begin{aligned}
& V_{\theta}=\varepsilon^{\mathrm{T}}\left(\Delta_{\theta_{0}}-\Delta_{\theta}\right) \varepsilon=\varepsilon^{\mathrm{T}}\left(\Pi_{\theta_{0}}-\Pi_{\theta}\right) \varepsilon+\varepsilon^{\mathrm{T}}\left(\boldsymbol{P}_{\boldsymbol{M}_{\boldsymbol{X}_{\theta_{0}}} \boldsymbol{B}}-\boldsymbol{P}_{\boldsymbol{M}_{\boldsymbol{X}_{\theta}} \boldsymbol{B}}\right) \varepsilon=V_{\theta, 1}+V_{\theta, 2}, \\
& A_{\theta}=\left(\boldsymbol{X}_{\theta_{0}} \boldsymbol{\beta}\right)^{\mathrm{T}}\left(\boldsymbol{I}-\Delta_{\theta}\right)\left(\boldsymbol{X}_{\theta_{0}} \boldsymbol{\beta}\right)=\left(\boldsymbol{X}_{\theta_{0}} \boldsymbol{\beta}\right)^{\mathrm{T}}\left(\boldsymbol{I}-\Pi_{\theta}\right)\left(\boldsymbol{X}_{\theta_{0}} \boldsymbol{\beta}\right)-\left(\boldsymbol{X}_{\theta_{0}} \boldsymbol{\beta}\right)^{\mathrm{T}} \boldsymbol{P}_{\boldsymbol{M}_{\boldsymbol{X}_{\theta}} \boldsymbol{B}}\left(\boldsymbol{X}_{\theta_{0}} \boldsymbol{\beta}\right) \\
& =A_{\theta, 1}-A_{\theta, 2} \text {, } \\
& S_{\theta}^{\varepsilon}=\varepsilon^{\mathrm{T}}\left(\boldsymbol{I}-\Delta_{\theta}\right) \boldsymbol{X}_{\theta_{0}} \boldsymbol{\beta}=\boldsymbol{\varepsilon}^{\mathrm{T}}\left(\boldsymbol{I}-\Pi_{\theta}\right) \boldsymbol{X}_{\theta_{0}} \boldsymbol{\beta}-\boldsymbol{\varepsilon}^{\mathrm{T}} \boldsymbol{P}_{\boldsymbol{M}_{\boldsymbol{X}_{\theta}} \boldsymbol{B}} \boldsymbol{X}_{\theta_{0}} \boldsymbol{\beta}=S_{\theta, 1}^{\boldsymbol{\varepsilon}}-S_{\theta, 2}^{\varepsilon}, \\
& S_{\theta}^{\boldsymbol{g}}=\boldsymbol{g}^{\mathrm{T}}\left(\boldsymbol{I}-\Delta_{\theta}\right) \boldsymbol{X}_{\theta_{0}} \boldsymbol{\beta}=\boldsymbol{g}^{\mathrm{T}}\left(\boldsymbol{I}-\Pi_{\theta}\right) \boldsymbol{X}_{\theta_{0}} \boldsymbol{\beta}-\boldsymbol{g}^{\mathrm{T}} \boldsymbol{P}_{\boldsymbol{M}_{\boldsymbol{X}_{\theta}} \boldsymbol{B}} \boldsymbol{X}_{\theta_{0}} \boldsymbol{\beta}=S_{\theta, 1}^{\boldsymbol{g}}-S_{\theta, 2}^{\boldsymbol{g}},
\end{aligned}
$$

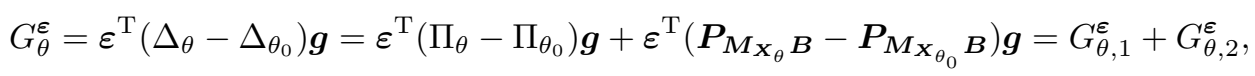

$$
\begin{aligned}
& G_{\theta}^{\boldsymbol{g}}=\boldsymbol{g}^{\mathrm{T}}\left(\Delta_{\theta}-\Delta_{\theta_{0}}\right) \boldsymbol{g}=\boldsymbol{g}^{\mathrm{T}}\left(\Pi_{\theta}-\Pi_{\theta_{0}}\right) \boldsymbol{g}+\boldsymbol{g}^{\mathrm{T}}\left(\boldsymbol{P}_{\boldsymbol{M}_{\boldsymbol{X}_{\theta}} \boldsymbol{B}}-\boldsymbol{P}_{\boldsymbol{M}_{\boldsymbol{X}_{\theta_{0}}} \boldsymbol{B}}\right) \boldsymbol{g}=G_{\theta, 1}^{\boldsymbol{g}}+G_{\theta, 2}^{\boldsymbol{g}} \text {. }
\end{aligned}
$$

接下来首先分析 $V_{\theta} 、 A_{\theta} 、 S_{\theta}^{\varepsilon} 、 S_{\theta}^{g} 、 G_{\theta}^{\varepsilon}$ 和 $G_{\theta}^{g}$, 然后综合起来证明不等式 (A.1).

为了分析 $A_{\theta} 、 S_{\theta}^{\varepsilon}$ 和 $S_{\theta}^{g}$, 首先看这几项共有的一项 $\left(\boldsymbol{I}-\Pi_{\theta}\right)\left(\boldsymbol{X}_{\theta_{0}} \boldsymbol{\beta}\right)$,

$$
\begin{aligned}
&\left(\boldsymbol{I}-\Pi_{\theta}\right) \boldsymbol{X}_{\theta_{0}} \boldsymbol{\beta}=\left(\boldsymbol{I}-\boldsymbol{X}_{\theta} D_{\theta} \boldsymbol{X}_{\theta}^{\mathrm{T}}\right) \boldsymbol{X}_{\theta_{0}} \boldsymbol{\beta} \\
&=\left(\boldsymbol{I}-\left(\begin{array}{c}
D_{\theta} D_{\theta} \cdots \\
D_{\theta} \cdot \\
\vdots
\end{array}\right)\right)\left(\begin{array}{c}
m(1) \\
\vdots \\
m\left(\theta_{0}\right) \\
m(1) \\
\vdots
\end{array}\right) \\
&=\left(\eta_{1, T}, \ldots, \eta_{\theta^{\times}, T}, \eta_{1, T}, \ldots, \eta_{\theta^{\times}, T}, \ldots\right)^{\mathrm{T}}, \\
& \eta_{s, T}=m(s)-\frac{1}{K_{s_{\theta}, T}^{[\theta]}} \sum_{k=1}^{K_{s_{\theta}, T}^{[\theta}} m\left((k-1) \theta+s_{\theta}\right),
\end{aligned}
$$


其中 $s=1, \ldots, \theta^{\times}, s_{\theta}=s-\theta\left\lfloor\frac{s}{\theta}\right\rfloor, \theta^{\times}$是 $\theta$ 和 $\theta_{0}$ 的最小公倍数. 为了方便证明, 将 $\eta_{s, T}$ 写成如下形式:

$$
\eta_{s, T}=\zeta_{s}+R_{s, T}
$$

其中 $R_{s, T}=R_{1, s, T}+R_{2, s, T}$, 并且

$$
\begin{aligned}
& \zeta_{s}=m(s)-\frac{1}{\theta_{0}} \sum_{k=1}^{\theta_{0}} m\left((k-1) \theta+s_{\theta}\right), \\
& R_{1, s, T}=\left(1-\frac{\theta_{0}}{K_{s_{\theta}, T}^{(\theta]}}\left\lfloor\frac{K_{s_{\theta}, T}^{[\theta]}}{\theta_{0}}\right\rfloor\right) \frac{1}{\theta_{0}} \sum_{k=1}^{\theta_{0}} m\left((k-1) \theta+s_{\theta}\right), \\
& R_{2, s, T}=-\frac{1}{K_{s_{\theta}, T}^{[\theta]}} \sum_{k=\theta_{0}\left\lfloor K_{s_{\theta}, T}^{[\theta]} / \theta_{0}\right\rfloor}^{K_{s_{\theta}, T}^{[\theta]}} m\left((k-1) \theta+s_{\theta}\right) .
\end{aligned}
$$

根据文献 [14, 定理 1 的证明] 知

$$
\left|R_{s, T}\right| \leqslant \frac{C \theta_{0}}{K_{s_{\theta}, T}^{[\theta]}}
$$

引理 $\mathbf{A . 2}$ 存在一个自然数 $T_{0}$, 使得当 $T \geqslant T_{0}$ 时, 以下结论恒成立:

情形 $1 \quad \theta \neq \theta_{0}$ 且 $\theta$ 不是 $\theta_{0}$ 的倍数, 我们有

$$
A_{\theta} \geqslant c\left(\frac{n T}{\theta}\right), \quad \mathrm{P}\left(\left|S_{\theta}^{\varepsilon}\right|>\nu_{T} \sqrt{\frac{n T}{\theta}}\right) \leqslant C \nu_{T}^{-2}, \quad\left|S_{\theta}^{g}\right| \leqslant C n,
$$

其中 $n=\sharp \mathcal{S}, \mathcal{S}=\left\{s \mid s \in\left\{1, \ldots, \theta^{\times}\right\}, \zeta_{s} \neq 0\right\}, \nu_{T}$ 是一列缓慢发散到正无穷的正数序列.

情形 $2 \theta \neq \theta_{0}$ 且 $\theta$ 是 $\theta_{0}$ 的倍数, 我们有

$$
A_{\theta}=0, \quad S_{\theta}^{\varepsilon}=0, \quad S_{\theta}^{g}=0 .
$$

证明 根据文献 $\left[14\right.$, 引理 A.4] 可知, 存在一个自然数 $T_{0}$ 使得 $T \geqslant T_{0}$ 时, 以下结论恒成立:

情形 $1 \quad \theta \neq \theta_{0}$ 且 $\theta$ 不是 $\theta_{0}$ 的倍数, 我们有

$$
A_{\theta, 1} \geqslant c\left(\frac{n T}{\theta}\right), \quad \mathrm{P}\left(\left|S_{\theta, 1}^{\varepsilon}\right|>\nu_{T} \sqrt{\frac{n T}{\theta}}\right) \leqslant C \nu_{T}^{-2}, \quad\left|S_{\theta, 1}^{g}\right| \leqslant C n
$$

其中 $n=\sharp \mathcal{S}, \mathcal{S}=\left\{s \mid s \in\left\{1, \ldots, \theta^{\times}\right\}, \zeta_{s} \neq 0\right\}, \nu_{T}$ 是一列缓慢发散到正无穷的正数序列.

情形 $2 \theta \neq \theta_{0}$ 且 $\theta$ 是 $\theta_{0}$ 的倍数, 我们有

$$
A_{\theta, 1}=0, \quad S_{\theta, 1}^{\varepsilon}=0, \quad S_{\theta, 1}^{g}=0 .
$$

我们可以证明, 当 $\theta \neq \theta_{0}$ 且 $\theta$ 是 $\theta_{0}$ 的倍数时, $\boldsymbol{P}_{\boldsymbol{M}_{\boldsymbol{X}_{\theta}} \boldsymbol{B}}\left(\boldsymbol{X}_{\theta_{0}} \boldsymbol{\beta}\right)=\mathbf{0}$. 所以存在一个自然数 $T_{0}$, 使得 当 $T \geqslant T_{0}$ 时, 以下结论恒成立:

当 $\theta \neq \theta_{0}$ 且 $\theta$ 是 $\theta_{0}$ 的倍数时, $A_{\theta}=0, S_{\theta}^{\varepsilon}=0, S_{\theta}^{\boldsymbol{g}}=0$. 
现在只需证明 $\theta$ 不是 $\theta_{0}$ 的倍数时, 情形 1 的结论成立即可. 令 $A_{\theta}=A_{\theta, 1}-A_{\theta, 2}>(1-\delta) A_{\theta, 1}$, 其 中 $0<\delta<1$ 是一个很小的常数.

实际上 $A_{\theta, 2}<A_{\theta, 1}$, 这是因为 $\mathcal{C}\left(\left(\boldsymbol{I}-\Pi_{\theta}\right) \boldsymbol{B}\right) \subset \mathcal{C}\left(\left(\boldsymbol{I}-\Pi_{\theta}\right)\right)$, 而 $\boldsymbol{P}_{\boldsymbol{M}_{\boldsymbol{X}_{\theta}} \boldsymbol{B}}$ 和 $\boldsymbol{I}-\Pi_{\theta}$ 分别是这两个 空间的垂直投影矩阵. 根据投影矩阵的性质, 可以得到

$$
\begin{aligned}
& A_{\theta, 2}=\left(\boldsymbol{X}_{\theta_{0}} \boldsymbol{\beta}\right)^{\mathrm{T}} \boldsymbol{P}_{\boldsymbol{M}_{\boldsymbol{X}_{\theta}} \boldsymbol{B}}\left(\boldsymbol{X}_{\theta_{0}} \boldsymbol{\beta}\right)=\left(\boldsymbol{X}_{\theta_{0}} \boldsymbol{\beta}\right)^{\mathrm{T}} \boldsymbol{Q}\left(\begin{array}{cc}
\boldsymbol{I}_{\boldsymbol{N}_{0}} \mathbf{0} \\
\mathbf{0} & \mathbf{0}
\end{array}\right) \boldsymbol{Q}^{\mathrm{T}}\left(\boldsymbol{X}_{\theta_{0}} \boldsymbol{\beta}\right), \\
& A_{\theta, 1}=\left(\boldsymbol{X}_{\theta_{0}} \boldsymbol{\beta}\right)^{\mathrm{T}} \boldsymbol{Q} \boldsymbol{I}_{T} \boldsymbol{Q}^{\mathrm{T}}\left(\boldsymbol{X}_{\theta_{0}} \boldsymbol{\beta}\right)>c \frac{n T}{\theta},
\end{aligned}
$$

其中 $\boldsymbol{Q} \boldsymbol{Q}^{\mathrm{T}}=\boldsymbol{Q}^{\mathrm{T}} \boldsymbol{Q}=\boldsymbol{I}, \boldsymbol{N}_{0}<\min \left(\boldsymbol{N}_{T}, T-\theta\right), T \gg \boldsymbol{N}_{0}$.

所以, $A_{\theta} \geqslant C \frac{n T}{\theta}$. 同理可得

$$
\begin{aligned}
& \mathrm{P}\left(\left|S_{\theta}^{\varepsilon}\right|>\nu_{T} \sqrt{\frac{n T}{\theta}}\right) \leqslant \mathrm{P}\left(|| S_{\theta, 1}^{\varepsilon}|+| S_{\theta, 2}^{\varepsilon}||>\nu_{T} \sqrt{\frac{n T}{\theta}}\right) \leqslant \mathrm{P}\left(2\left|S_{\theta, 1}^{\varepsilon}\right|>\nu_{T} \sqrt{\frac{n T}{\theta}}\right) \leqslant C \nu_{T}^{-2}, \\
& \left|S_{\theta}^{\boldsymbol{g}}\right|=\left|S_{\theta, 1}^{\boldsymbol{g}}-S_{\theta, 2}^{\boldsymbol{g}}\right| \leqslant\left|S_{\theta, 1}^{\boldsymbol{g}}\right|+\left|S_{\theta, 2}^{\boldsymbol{g}}\right| \leqslant 2\left|S_{\theta, 1}^{\boldsymbol{g}}\right| \leqslant C n .
\end{aligned}
$$

引理 A.2 证毕.

引理 $\mathbf{A . 3}$ 存在一个自然数 $T_{0}$, 使得当 $T \geqslant T_{0}$ 时, $\left|G_{\theta}^{g}\right| \leqslant C T^{1 / 5}, \mathrm{P}\left(\left|G_{\theta}^{\varepsilon}\right|>\nu_{T} N_{T}\right) \leqslant C \nu_{T}^{-2}$.

证明 根据文献 $\left[14\right.$, 引理 A.5] 可知, 存在一个自然数 $T_{0}$, 使得当 $T \geqslant T_{0}$ 时, $\left|G_{\theta, 1}^{g}\right| \leqslant C, \mathrm{P}\left(\left|G_{\theta, 1}^{\varepsilon}\right|\right.$ $\left.>\nu_{T}\right) \leqslant C \nu_{T}^{-2}$. 前面已经得到投影矩阵 $\Delta_{\theta}=\Pi_{\theta}+\left(\boldsymbol{I}-\Pi_{\theta}\right) \boldsymbol{C}_{\theta}\left(\boldsymbol{I}-\Pi_{\theta}\right)$. 因为投影矩阵 $\Pi_{\theta}$ 的元素有 $T^{2}-T^{2} / \theta^{2}$ 个 0 和 $T^{2} / \theta^{2}$ 个 $1 / K_{s, T}^{[\theta]}$, 而 $K_{s, T}^{[\theta]}=O(T / \theta)$, 则 $1 / K_{s, T}^{[\theta]}=O(\theta / T)=o(1)$, 所以,

$$
\left(\boldsymbol{I}-\Pi_{\theta}\right) \boldsymbol{C}_{\theta}\left(\boldsymbol{I}-\Pi_{\theta}\right)=\boldsymbol{B}\left(\boldsymbol{B}^{\mathrm{T}} \boldsymbol{B}\right)^{-1} \boldsymbol{B}^{\mathrm{T}}+o_{p}(1) .
$$

所以,

$$
\begin{aligned}
&\left|G_{\theta, 2}^{\boldsymbol{g}}\right|=\left|\boldsymbol{g}^{\mathrm{T}}\left(\boldsymbol{P}_{\boldsymbol{M}_{\boldsymbol{X}_{\theta}} \boldsymbol{B}}-\boldsymbol{P}_{\boldsymbol{M}_{\boldsymbol{X}_{\theta_{0}}} \boldsymbol{B}}\right) \boldsymbol{g}\right| \\
&=\left|(\boldsymbol{g}-\boldsymbol{B} \boldsymbol{\alpha})^{\mathrm{T}}\left(\boldsymbol{P}_{\boldsymbol{M}_{\boldsymbol{X}_{\theta}} \boldsymbol{B}}-\boldsymbol{P}_{\boldsymbol{M}_{\boldsymbol{X}_{\theta_{0}}} \boldsymbol{B}}\right)(\boldsymbol{g}-\boldsymbol{B} \boldsymbol{\alpha})\right| \\
& \leqslant \sum_{i}^{T} C N_{T}^{-4} \leqslant C T^{1 / 5}, \\
&\left|G_{\theta}^{\boldsymbol{g}}\right|=\left|G_{\theta, 1}^{\boldsymbol{g}}\right|+\left|G_{\theta, 2}^{\boldsymbol{g}}\right| \leqslant C T^{1 / 5}, \\
&\left|G_{\theta, 2}^{\boldsymbol{\varepsilon}}\right|=\left|\boldsymbol{\varepsilon}^{\mathrm{T}}\left(\boldsymbol{P}_{\boldsymbol{M}_{\boldsymbol{X}_{\theta}} \boldsymbol{B}}-\boldsymbol{P}_{\boldsymbol{M}_{\boldsymbol{X}_{\theta_{0}}} \boldsymbol{B}}\right) \boldsymbol{g}\right| \\
&=\left|\varepsilon^{\mathrm{T}}\left(\boldsymbol{P}_{\boldsymbol{M}_{\boldsymbol{X}_{\theta}} \boldsymbol{B}}-\boldsymbol{P}_{\boldsymbol{M}_{\boldsymbol{X}_{\theta_{0}}} \boldsymbol{B}}\right)\left(\boldsymbol{g}-\boldsymbol{B} \boldsymbol{\alpha}^{*}\right)\right| \\
& \leqslant\left|\sum_{i}^{T} C N_{T}^{-2} \varepsilon_{t}\right|, \\
& \mathrm{P}\left(\left|G_{\theta, 2}^{\boldsymbol{\varepsilon}}\right|>\nu_{T} N_{T}\right) \leqslant \mathrm{P}\left(\left|\sum_{i}^{T} C N_{T}^{-2} \varepsilon_{t}\right|>\nu_{T} N_{T}\right) \leqslant C \nu_{T}^{-2} .
\end{aligned}
$$

所以, $\mathrm{P}\left(\left|G_{\theta}^{\varepsilon}\right|>\nu_{T} N_{T}\right) \leqslant \mathrm{P}\left(\left(\left|G_{\theta, 1}^{\varepsilon}\right|+\left|G_{\theta, 2}^{\varepsilon}\right|\right)>\nu_{T} N_{T}\right) \leqslant C \nu_{T}^{-2}$. 引理 A.3 证毕. 
最后来看 $V_{\theta}=V_{\theta, 1}+V_{\theta, 2}$, 其中

$$
\begin{aligned}
& V_{\theta, 1}=\varepsilon^{\mathrm{T}}\left(\Pi_{\theta_{0}}-\Pi_{\theta}\right) \varepsilon \\
& =\varepsilon^{\mathrm{T}}\left(\left(\begin{array}{ccc}
D_{\theta_{0}} & D_{\theta_{0}} & \cdots \\
D_{\theta_{0}} & \ddots & \\
\vdots & &
\end{array}\right)-\left(\begin{array}{ccc}
D_{\theta} & D_{\theta} & \cdots \\
D_{\theta} & \ddots & \\
\vdots &
\end{array}\right)\right) \varepsilon \\
& =\sum_{l=1}^{T} \frac{1}{K_{l_{\theta_{0}}, T}^{\left[\theta_{\theta}\right]}} \sum_{k=1}^{K_{l_{\theta_{0}}}^{\left[\theta_{0}\right]}} \varepsilon_{(k-1) \theta_{0}+l_{\theta_{0}}, T^{\varepsilon_{l}, T}}-\sum_{l=1}^{T} \frac{1}{K_{l_{\theta}, T}^{[\theta]}} \sum_{k=1}^{K_{l_{\theta} T}^{[\theta]}} \varepsilon_{(k-1) \theta+l_{\theta}, T^{\varepsilon_{l, T}}} \\
& =: V_{\theta, 11}+V_{\theta, 12} \text {, }
\end{aligned}
$$

其中 $l_{\theta}=l-\theta\lfloor l / \theta\rfloor$, 并且 $\left|V_{\theta, 2}\right|<\left|V_{\theta, 1}\right|$. 令 $\nu_{T}=\left(\kappa_{T} \Theta_{T}\right)^{1 / 2}$, 在情形 1 下,

$$
\begin{aligned}
\mathrm{P}\left(Q\left(\theta, \lambda_{T}\right) \leqslant Q\left(\theta_{0}, \lambda_{T}\right)\right)= & \mathrm{P}\left(V_{\theta} \leqslant-A_{\theta}-2 S_{\theta}^{\varepsilon}-2 S_{\theta}^{g}+2 G_{\theta}^{\varepsilon}+G_{\theta}^{g}+\lambda_{T} N_{T}\left(\theta_{0}-\theta\right)\right) \\
\leqslant & \mathrm{P}\left(V_{\theta} \leqslant-A_{\theta}-2 S_{\theta}^{\varepsilon}-2 S_{\theta}^{g}+2 G_{\theta}^{\varepsilon}+G_{\theta}^{g}+\lambda_{T} N_{T}\left(\theta_{0}-\theta\right)\right. \\
& \left.\left|S_{\theta}^{\varepsilon}\right| \leqslant \nu_{T} \sqrt{\frac{n T}{\theta}},\left|G_{\theta}^{\varepsilon}\right| \leqslant \nu_{T} N_{T}\right)+\mathrm{P}\left(\left|S_{\theta}^{\varepsilon}\right|>\nu_{T} \sqrt{\frac{n T}{\theta}}\right)+\mathrm{P}\left(\left|G_{\theta}^{\varepsilon}\right|>\nu_{T} N_{T}\right) \\
\leqslant & \mathrm{P}\left(V_{\theta} \leqslant-A_{\theta}+C \nu_{T} \sqrt{\frac{n T}{\theta}}+C \nu_{T} N_{T}+\lambda_{T} N_{T}\left(\theta_{0}-\theta\right)\right)+C \nu_{T}^{-2} .
\end{aligned}
$$

选择 $\lambda_{T}$ 使得 $\left(\lambda_{T} N_{T}\right) / T \rightarrow 0$, 则

$$
\begin{aligned}
\mathrm{P}\left(Q\left(\theta, \lambda_{T}\right) \leqslant Q\left(\theta_{0}, \lambda_{T}\right)\right) & \leqslant \mathrm{P}\left(V_{\theta} \leqslant-C_{1} \frac{n T}{\theta}\right)+C \nu_{T}^{-2} \\
& \leqslant \mathrm{P}\left(V_{\theta} \leqslant-C_{1} \frac{T}{\Theta_{T}}\right)+C \nu_{T}^{-2} \\
\mathrm{P}\left(V_{\theta} \leqslant-C_{1} \frac{T}{\Theta_{T}}\right) & =\mathrm{P}\left(V_{\theta, 1}+V_{\theta, 2} \leqslant-C_{1} \frac{T}{\Theta_{T}}\right) \\
& \leqslant \mathrm{P}\left(\left|V_{\theta, 1}\right|+\left|V_{\theta, 2}\right| \geqslant C_{1} \frac{T}{\Theta_{T}}\right) \\
& \leqslant \mathrm{P}\left(2\left|V_{\theta, 1}\right| \geqslant C_{1} \frac{T}{\Theta_{T}}\right) \\
& \leqslant C\left(\kappa_{T} \Theta_{T}\right)^{-1},
\end{aligned}
$$

上式最后一步是根据文献 [14, 定理 1 的证明] 得到的. 所以, 在情形 1 下, $\mathrm{P}\left(Q\left(\theta, \lambda_{T}\right) \leqslant Q\left(\theta_{0}, \lambda_{T}\right)\right)$ $\leqslant C\left(\kappa_{T} \Theta_{T}\right)^{-1}$. 相应地, 在情形 2 下, 选择 $\lambda_{T}$ 使得 $\nu_{T} / \lambda_{T} \rightarrow 0$, 则

$$
\begin{aligned}
\mathrm{P}\left(Q\left(\theta, \lambda_{T}\right) \leqslant Q\left(\theta_{0}, \lambda_{T}\right)\right) & =\mathrm{P}\left(V_{\theta} \leqslant 2 G_{\theta}^{\varepsilon}+G_{\theta}^{g}+\lambda_{T} N_{T}\left(\theta_{0}-\theta\right)\right) \\
& \leqslant \mathrm{P}\left(V_{\theta} \leqslant 2 G_{\theta}^{\varepsilon}+G_{\theta}^{g}+\lambda_{T} N_{T}\left(\theta_{0}-\theta\right),\left|G_{\theta}^{\varepsilon}\right| \leqslant \nu_{T} N_{T}\right)+C \nu_{T}^{-2} \\
& \leqslant \mathrm{P}\left(V_{\theta} \leqslant C \nu_{T} N_{T}+C N_{T}+\lambda_{T} N_{T}\left(\theta_{0}-\theta\right)\right)+C \nu_{T}^{-2}
\end{aligned}
$$




$$
\begin{aligned}
& \leqslant \mathrm{P}\left(V_{\theta} \leqslant C \nu_{T} N_{T}+\lambda_{T} N_{T}\left(\theta_{0}-\theta\right)\right)+C \nu_{T}^{-2} \\
& \leqslant \mathrm{P}\left(2\left|V_{\theta, 1}\right| \geqslant C_{2} \lambda_{T} N_{T}\right)+C \nu_{T}^{-2} \\
& \leqslant C\left(\frac{\Theta_{T} \log T}{\lambda_{T} N_{T}}\right)^{2}
\end{aligned}
$$

上式最后一步是根据文献 [14, 定理 1 的证明] 得到的. 选择 $\lambda_{T}$ 使得 $\lambda_{T} N_{T} \geqslant \tau_{T}(\log T) \Theta_{T}^{3 / 2}, \tau_{T}$ 是一 个缓慢发散到正无穷的序列, 例如 $\tau_{T}=\log \log T$, 那么在情形 2 下也得到了

$$
\mathrm{P}\left(Q\left(\theta, \lambda_{T}\right) \leqslant Q\left(\theta_{0}, \lambda_{T}\right)\right) \leqslant C\left(\kappa_{T} \Theta_{T}\right)^{-1}
$$

\section{附录 A.2 定理 3.2 的证明}

记 $\tilde{m}$ 是在周期 $\theta_{0}$ 已知的情形下 $m$ 的估计, $\left(\tilde{m}(1), \ldots, \tilde{m}\left(\theta_{0}\right)\right)=\hat{\boldsymbol{\beta}}_{\theta_{0}}$, 并且 $\tilde{m}\left(s+k \theta_{0}\right)=\tilde{m}(s)$, $s=1, \ldots, \theta_{0}, k \in \mathbb{N}$, 则有

$$
\sqrt{T}(\hat{m}(t)-m(t))=\sqrt{T}(\hat{m}(t)-\tilde{m}(t))+\sqrt{T}(\tilde{m}(t)-m(t)) .
$$

对于任意 $\delta>0$, 有

$$
\mathrm{P}(|\sqrt{T}(\hat{m}(t)-\tilde{m}(t))|>\delta) \leqslant \mathrm{P}\left(|\sqrt{T}(\hat{m}(t)-\tilde{m}(t))|>\delta, \hat{\theta}=\theta_{0}\right)+\mathrm{P}\left(\hat{\theta} \neq \theta_{0}\right) .
$$

注意到, 当 $\hat{\theta}=\theta_{0}$ 时, $\hat{m}(t)=\tilde{m}(t)$, 由定理 3.1 可知, $\mathrm{P}\left(\hat{\theta} \neq \theta_{0}\right)=o(1)$, 所以,

$$
\sqrt{T}(\hat{m}(t)-m(t))=\sqrt{T}(\tilde{m}(t)-m(t))+o_{p}(1) .
$$

我们可以证明

$$
\begin{aligned}
\hat{\boldsymbol{\beta}}_{\theta_{0}} & =\left(\boldsymbol{X}_{\theta_{0}}^{\mathrm{T}}\left(\boldsymbol{I}-\boldsymbol{B}\left(\boldsymbol{B}^{\mathrm{T}} \boldsymbol{B}\right)^{-1} \boldsymbol{B}^{\mathrm{T}}\right) \boldsymbol{X}_{\theta_{0}}\right)^{-1} \boldsymbol{X}_{\theta_{0}}^{\mathrm{T}}\left(\boldsymbol{I}-\boldsymbol{B}\left(\boldsymbol{B}^{\mathrm{T}} \boldsymbol{B}\right)^{-1} \boldsymbol{B}^{\mathrm{T}}\right) \boldsymbol{Y} \\
& =\left(\boldsymbol{X}_{\theta_{0}}^{\mathrm{T}}\left(\boldsymbol{I}-\boldsymbol{P}_{B}\right) \boldsymbol{X}_{\theta_{0}}\right)^{-1} \boldsymbol{X}_{\theta_{0}}^{\mathrm{T}}\left(\boldsymbol{I}-\boldsymbol{P}_{B}\right) \boldsymbol{Y} .
\end{aligned}
$$

当周期已知时, 在普通的部分线性模型下, 我们可以证明当 $N / T+N^{-4}=o\left(T^{-1 / 2}\right)$, 即非线性部 分的估计的均方误差相对可忽略时, $\sqrt{T}\left(\tilde{\boldsymbol{\beta}}_{\theta_{0}}-\boldsymbol{\beta}_{\theta_{0}}\right)$ 依分布收玫到正态分布, 并且 $\sqrt{T}(\tilde{m}(t)-m(t))$ 依 分布收玫到正态分布. 所以 $\sqrt{T}(\hat{m}(t)-m(t))$ 也依分布收玫到正态分布, 证明过程与文献 [17, 定理 4.1] 和 [19, 定理 1] 的证明相似.

我们来看在周期已知的情形下线性部分收玫速度的具体证明过程. 注意到, 系数估计可以分解成 以下两部分:

$$
\begin{aligned}
\tilde{\boldsymbol{\beta}}_{\theta_{0}}-\boldsymbol{\beta}_{\theta_{0}} & =\left(\boldsymbol{X}_{\theta_{0}}^{\mathrm{T}}\left(\boldsymbol{I}-\boldsymbol{P}_{B}\right) \boldsymbol{X}_{\theta_{0}}\right)^{-1} \boldsymbol{X}_{\theta_{0}}^{\mathrm{T}}\left(\boldsymbol{I}-\boldsymbol{P}_{B}\right) \boldsymbol{Y}-\boldsymbol{\beta} \\
& =\left(\boldsymbol{X}_{\theta_{0}}^{\mathrm{T}}\left(\boldsymbol{I}-\boldsymbol{P}_{B}\right) \boldsymbol{X}_{\theta_{0}}\right)^{-1} \boldsymbol{X}_{\theta_{0}}^{\mathrm{T}}\left(\boldsymbol{I}-\boldsymbol{P}_{B}\right)(\boldsymbol{g}+\boldsymbol{\varepsilon}) \\
& =\left(\boldsymbol{X}_{\theta_{0}}^{\mathrm{T}}\left(\boldsymbol{I}-\boldsymbol{P}_{B}\right) \boldsymbol{X}_{\theta_{0}}\right)^{-1} \boldsymbol{X}_{\theta_{0}}^{\mathrm{T}}\left(\boldsymbol{I}-\boldsymbol{P}_{B}\right) \boldsymbol{g}+\left(\boldsymbol{X}_{\theta_{0}}^{\mathrm{T}}\left(\boldsymbol{I}-\boldsymbol{P}_{B}\right) \boldsymbol{X}_{\theta_{0}}\right)^{-1} \boldsymbol{X}_{\theta_{0}}^{\mathrm{T}}\left(\boldsymbol{I}-\boldsymbol{P}_{B}\right) \varepsilon .
\end{aligned}
$$


引理 A.4 $T N_{T}^{-1} \boldsymbol{P}_{B}$ 和 $T N_{T}^{-1}\left(\boldsymbol{B}^{\mathrm{T}} \boldsymbol{B}\right)^{-1}$ 是依概率有界的, 即 $T N_{T}^{-1} \boldsymbol{P}_{B}$ 和 $T N_{T}^{-1}\left(\boldsymbol{B}^{\mathrm{T}} \boldsymbol{B}\right)^{-1}$ 的每 个元素是 $O_{P}(1)$.

引理 A.4 的证明参见文献 $[23,24]$.

引理 $\mathbf{A . 5}$ 给定 $T=t, \tilde{\boldsymbol{x}}_{i}(t)=\mathrm{E}\left(\boldsymbol{x}_{i} \mid t\right), \boldsymbol{e}_{i}=\boldsymbol{x}_{i}-\tilde{\boldsymbol{x}}_{i}(t), \Phi=\left(\sigma_{i j}\right)_{\theta_{0} \times \theta_{0}}, 1 \leqslant i, j \leqslant \theta_{0}$, 其中

$$
\sigma_{i j}=\operatorname{Cov}\left(\boldsymbol{x}_{i}-\tilde{\boldsymbol{x}}_{i}(T), \boldsymbol{x}_{j}-\tilde{\boldsymbol{x}}_{j}(T)\right)
$$

则下式成立:

$$
\frac{\boldsymbol{X}_{\theta_{0}}^{\mathrm{T}}\left(\boldsymbol{I}-\boldsymbol{P}_{B}\right) \boldsymbol{X}_{\theta_{0}}}{T} \rightarrow \Phi, \quad T \rightarrow \infty
$$

证明 注意到

$$
\begin{aligned}
\begin{aligned}
\left(\boldsymbol{X}_{\theta_{0}}^{\mathrm{T}}\left(\boldsymbol{I}-\boldsymbol{P}_{B}\right) \boldsymbol{X}_{\theta_{0}}\right)_{i j}= & \left(\boldsymbol{e}_{i}+\tilde{\boldsymbol{x}}_{i}(T)\right)^{\mathrm{T}}\left(\boldsymbol{I}-\boldsymbol{P}_{B}\right)\left(\boldsymbol{e}_{i}+\tilde{\boldsymbol{x}}_{i}(T)\right) \\
= & \boldsymbol{e}_{i}^{\mathrm{T}}\left(\boldsymbol{I}-\boldsymbol{P}_{B}\right) \boldsymbol{e}_{j}+\boldsymbol{e}_{i}^{\prime}\left(\boldsymbol{I}-\boldsymbol{P}_{B}\right) \theta_{j}(T) \\
& +\tilde{\boldsymbol{x}}_{i}(T)^{\mathrm{T}}\left(\boldsymbol{I}-\boldsymbol{P}_{B}\right) \boldsymbol{e}_{j}+\tilde{\boldsymbol{x}}_{i}(T)^{\mathrm{T}}\left(\boldsymbol{I}-\boldsymbol{P}_{B}\right) \tilde{\boldsymbol{x}}_{j}(T), \\
\mathrm{E}\left(\left|\boldsymbol{e}_{i}^{\prime} \boldsymbol{P}_{B} \boldsymbol{e}_{i}\right| \mid \boldsymbol{P}_{B}\right)= & \mathrm{E}\left(\boldsymbol{e}_{i}^{\prime} \boldsymbol{P}_{B} \boldsymbol{e}_{i} \mid \boldsymbol{P}_{B}\right)=\mathrm{E}\left(\operatorname{tr}\left(\boldsymbol{P}_{B} \boldsymbol{e}_{i} \boldsymbol{e}_{i}^{\prime}\right) \mid \boldsymbol{P}_{B}\right) \\
= & O_{p}(1) \operatorname{tr}\left(\boldsymbol{P}_{B} \mathrm{E}\left(\boldsymbol{e}_{i} \boldsymbol{e}_{i}^{\prime}\right)\right)=\left[\operatorname{Var}\left(\boldsymbol{x}_{i}-\tilde{\boldsymbol{x}}_{i}(T)\right)\right] N_{T} O_{p}(1) \\
= & o_{p}(T), \\
\mathrm{E}\left(\left|\boldsymbol{e}_{i}^{\prime} \boldsymbol{P}_{B} \boldsymbol{e}_{j}\right| \mid \boldsymbol{P}_{B}\right) \leqslant & \frac{\mathrm{E}\left(\boldsymbol{e}_{i}^{\prime} \boldsymbol{P}_{B} \boldsymbol{e}_{i} \mid \boldsymbol{P}_{B}\right)+\mathrm{E}\left(\boldsymbol{e}_{j}^{\prime} \boldsymbol{P}_{B} \boldsymbol{e}_{j} \mid \boldsymbol{P}_{B}\right)}{2}=o_{p}(T) .
\end{aligned}
\end{aligned}
$$

所以根据大数定律, 有

$$
\begin{aligned}
& \frac{\boldsymbol{e}_{i}^{\prime} \boldsymbol{e}_{j}}{T} \stackrel{p}{\longrightarrow} \sigma_{i j}, \quad T \rightarrow \infty, \\
& \frac{\boldsymbol{e}_{i}^{\prime}\left(\boldsymbol{I}-\boldsymbol{P}_{B}\right) \boldsymbol{e}_{j}}{T} \stackrel{p}{\longrightarrow} \sigma_{i j}, \quad T \rightarrow \infty .
\end{aligned}
$$

同时我们可以证明

$$
\begin{aligned}
& \frac{\boldsymbol{e}_{i}^{\prime}\left(\boldsymbol{I}-\boldsymbol{P}_{B}\right) \tilde{\boldsymbol{x}}_{j}(T)}{T}=\frac{N^{-2}}{\sqrt{T}}=o_{p}(1) \\
& \frac{\tilde{\boldsymbol{x}}_{i}(T)^{\mathrm{T}}\left(\boldsymbol{I}-\boldsymbol{P}_{B}\right) \tilde{\boldsymbol{x}}_{j}(T)}{T}=\tilde{\boldsymbol{x}}_{i}(T)^{\mathrm{T}} \frac{\left(\boldsymbol{I}-\boldsymbol{P}_{B}\right)\left(\boldsymbol{I}-\boldsymbol{P}_{B}\right) \tilde{\boldsymbol{x}}_{j}(T)}{T}=O_{p}\left(N^{-4}\right)=o_{p}(1) .
\end{aligned}
$$

引理 A.5 证毕.

由于 $\left(\boldsymbol{I}-\boldsymbol{P}_{B}\right) \boldsymbol{g}=\left(\boldsymbol{I}-\boldsymbol{P}_{B}\right)(\boldsymbol{g}-\boldsymbol{B} \boldsymbol{\alpha}+\boldsymbol{B} \boldsymbol{\alpha})=\left(\boldsymbol{I}-\boldsymbol{P}_{B}\right)(\boldsymbol{g}-\boldsymbol{B} \boldsymbol{\alpha})$, 故 $\left|\left(\left(\boldsymbol{I}-\boldsymbol{P}_{B}\right) \boldsymbol{g}\right)_{i}\right|=O\left(N^{-2}\right)$, $1 \leqslant i \leqslant T$. 注意到

$$
\begin{aligned}
& \sqrt{T}\left(\boldsymbol{X}_{\theta_{0}}^{\mathrm{T}}\left(\boldsymbol{I}-\boldsymbol{P}_{B}\right) \boldsymbol{X}_{\theta_{0}}\right)^{-1} \boldsymbol{X}_{\theta_{0}}^{\mathrm{T}}\left(\boldsymbol{I}-\boldsymbol{P}_{B}\right) \boldsymbol{g}=\left(\frac{\boldsymbol{X}_{\theta_{0}}^{\mathrm{T}}\left(\boldsymbol{I}-\boldsymbol{P}_{B}\right) \boldsymbol{X}_{\theta_{0}}}{T}\right)^{-1} T^{-1 / 2} \boldsymbol{X}_{\theta_{0}}^{\mathrm{T}}\left(\boldsymbol{I}-\boldsymbol{P}_{B}\right)\left(\boldsymbol{I}-\boldsymbol{P}_{B}\right) \boldsymbol{g}, \\
& \sqrt{T}\left(\boldsymbol{X}_{\theta_{0}}^{\mathrm{T}}\left(\boldsymbol{I}-\boldsymbol{P}_{B}\right) \boldsymbol{X}_{\theta_{0}}\right)^{-1} \boldsymbol{X}_{\theta_{0}}^{\mathrm{T}}\left(\boldsymbol{I}-\boldsymbol{P}_{B}\right) \boldsymbol{\varepsilon}=\left(\frac{\boldsymbol{X}_{\theta_{0}}^{\mathrm{T}}\left(\boldsymbol{I}-\boldsymbol{P}_{B}\right) \boldsymbol{X}_{\theta_{0}}}{T}\right)^{-1} T^{-1 / 2} \boldsymbol{X}_{\theta_{0}}^{\mathrm{T}}\left(\boldsymbol{I}-\boldsymbol{P}_{B}\right)\left(\boldsymbol{I}-\boldsymbol{P}_{B}\right) \boldsymbol{\varepsilon},
\end{aligned}
$$


而

$$
\begin{aligned}
\left(T^{-1 / 2} \boldsymbol{X}_{\theta_{0}}^{\mathrm{T}}\left(\boldsymbol{I}-\boldsymbol{P}_{B}\right)\left(\boldsymbol{I}-\boldsymbol{P}_{B}\right) \boldsymbol{g}\right)_{j} & =T^{-1 / 2}\left(\boldsymbol{e}_{j}+\tilde{\boldsymbol{x}}_{j}(T)\right)^{\mathrm{T}}\left(\boldsymbol{I}-\boldsymbol{P}_{B}\right)\left(\boldsymbol{I}-\boldsymbol{P}_{B}\right) \boldsymbol{g} \\
& =T^{-1 / 2} \boldsymbol{e}_{j}^{\mathrm{T}}\left(\boldsymbol{I}-\boldsymbol{P}_{B}\right)\left(\boldsymbol{I}-\boldsymbol{P}_{B}\right) \boldsymbol{g}+\tilde{\boldsymbol{x}}_{j}^{\mathrm{T}}(T)\left(\boldsymbol{I}-\boldsymbol{P}_{B}\right)\left(\boldsymbol{I}-\boldsymbol{P}_{B}\right) \boldsymbol{g} \\
& =O_{p}\left(T * T^{-1 / 2} N^{-2}\left(\frac{N}{T}\right)^{1 / 2}\right)+O\left(T * T^{-1 / 2} N^{-4}\right) \\
& =O_{p}\left(T^{1 / 2} N^{-2}\left(\frac{N}{T}\right)^{1 / 2}\right)+O\left(T^{1 / 2} N^{-4}\right),
\end{aligned}
$$

若内部节点数 $N$ 满足 $N / T+N^{-4}=o\left(T^{-1 / 2}\right)$, 则有

$$
\sqrt{T}\left(\boldsymbol{X}_{\theta_{0}}^{\mathrm{T}}\left(\boldsymbol{I}-\boldsymbol{P}_{B}\right) \boldsymbol{X}_{\theta_{0}}\right)^{-1} \boldsymbol{X}_{\theta_{0}}^{\mathrm{T}}\left(\boldsymbol{I}-\boldsymbol{P}_{B}\right) \boldsymbol{g}=o_{p}(1) .
$$

接下来证明

$$
T^{-1 / 2} \boldsymbol{X}_{\theta_{0}}^{\mathrm{T}}\left(\boldsymbol{I}-\boldsymbol{P}_{B}\right) \varepsilon \stackrel{d}{\longrightarrow} N\left(\mathbf{0}, \Sigma_{1}\right),
$$

其中 $\Sigma_{1}=\sum_{i, j=1}^{T} \operatorname{Cov}\left(\left(\boldsymbol{x}_{i}-\tilde{\boldsymbol{x}}_{i}(t)\right) \varepsilon_{i},\left(\boldsymbol{x}_{j}-\tilde{\boldsymbol{x}}_{j}(t)\right) \varepsilon_{j}\right) / T, \boldsymbol{x}_{t_{0}+(k-1) \theta_{0}}^{\mathrm{T}}$ 是 $\boldsymbol{X}_{\theta_{0}}$ 的行向量.

对于 (A.2) 中第二部分, 有

$$
\begin{aligned}
T^{-1 / 2} \boldsymbol{X}_{\theta_{0}}^{\mathrm{T}}\left(\boldsymbol{I}-\boldsymbol{P}_{B}\right) \boldsymbol{\varepsilon} & =T^{-1 / 2}(\boldsymbol{e}+\tilde{\boldsymbol{x}}(T))^{\mathrm{T}}\left(\boldsymbol{I}-\boldsymbol{P}_{B}\right)\left(\boldsymbol{I}-\boldsymbol{P}_{B}\right) \boldsymbol{\varepsilon} \\
& =T^{-1 / 2} \boldsymbol{e}^{\mathrm{T}}\left(\boldsymbol{I}-\boldsymbol{P}_{B}\right)\left(\boldsymbol{I}-\boldsymbol{P}_{B}\right) \boldsymbol{\varepsilon}+\tilde{\boldsymbol{x}}^{\mathrm{T}}(T)\left(\boldsymbol{I}-\boldsymbol{P}_{B}\right)\left(\boldsymbol{I}-\boldsymbol{P}_{B}\right) \boldsymbol{\varepsilon} \\
& =T^{-1 / 2} \boldsymbol{e}^{\mathrm{T}}\left(\boldsymbol{I}-\boldsymbol{P}_{B}\right) \boldsymbol{\varepsilon}+o_{p}(1) \\
& =T^{-1 / 2} \boldsymbol{e}^{\mathrm{T}} \boldsymbol{\varepsilon}-T^{-1 / 2} \boldsymbol{e}^{\mathrm{T}} \boldsymbol{P}_{B} \boldsymbol{\varepsilon}+o_{p}(1) \\
& =T^{-1 / 2} \boldsymbol{e}^{\mathrm{T}} \boldsymbol{\varepsilon}+o_{p}(1) \\
& \stackrel{d}{\longrightarrow} N\left(\mathbf{0}, \Sigma_{1}\right) .
\end{aligned}
$$

所以, 由 (A.2)-(A.5), 有

$$
\sqrt{T}\left(\tilde{\boldsymbol{\beta}}_{\theta_{0}}-\boldsymbol{\beta}_{\theta_{0}}\right) \stackrel{d}{\longrightarrow} N\left(\mathbf{0}, \Phi^{-1} \Sigma_{1} \Phi^{-1}\right),
$$

定理 3.2 得证.

\section{附录 A.3 定理 3.3 的证明}

我们可以证明

$$
\hat{\boldsymbol{\alpha}}=\left(\boldsymbol{B}^{\mathrm{T}} \boldsymbol{B}\right)^{-1} \boldsymbol{B}^{\mathrm{T}}\left(\boldsymbol{Y}-\boldsymbol{X}_{\theta_{0}} \hat{\boldsymbol{\beta}}\right) .
$$

定义 $\tilde{g}(u)$ 是周期序列 $m$ 已知时 $g$ 的不可行估计, 则

$$
\sup _{u \in[0,1]}|\hat{g}(u)-g(u)| \leqslant \sup _{u \in[0,1]}|\hat{g}(u)-\tilde{g}(u)|+\sup _{u \in[0,1]}|\tilde{g}(u)-g(u)| .
$$


根据定理 3.2 , 有

$$
\boldsymbol{\beta}-\hat{\boldsymbol{\beta}}=O_{p}\left(\frac{1}{\sqrt{T}}\right), \quad \sup _{u \in[0,1]}|\hat{g}(u)-\tilde{g}(u)|=\sup _{u \in[0,1]}\left|\boldsymbol{B}(u)^{\mathrm{T}}\left(\boldsymbol{B}^{\mathrm{T}} \boldsymbol{B}\right)^{-1} \boldsymbol{B}^{\mathrm{T}} \boldsymbol{X}_{\theta_{0}}(\boldsymbol{\beta}-\hat{\boldsymbol{\beta}})\right|=O_{p}\left(\frac{1}{\sqrt{T}}\right) .
$$

所以只需证明存在常数列 $\zeta_{0}\left(N_{T}\right)$ 满足 $\sup _{u \in[0,1]}\|\boldsymbol{B}(u)\| \leqslant \zeta_{0}\left(N_{T}\right)$ 且 $\zeta_{0}\left(N_{T}\right)^{2} N_{T} / T \rightarrow 0$, 则有

$$
\sup _{u \in[0,1]}|\tilde{g}(u)-g(u)|=O_{p}\left(\zeta_{0}(N)\left(\sqrt{\frac{N}{T}}+N^{-2}\right)\right) .
$$

仿照部分线性模型的证明 (参见文献 [19, 定理 2]), 我们将 $\tilde{g}$ 分解成方差项 $\tilde{g}^{V}(u)=\tilde{g}(u)-\mathrm{E}[\tilde{g}(u)]$ 和 偏差项 $\tilde{g}^{B}(u)=\mathrm{E}[\tilde{g}(u)]-g(u)$, 可以证明 $\left|\tilde{g}^{B}(u)\right|=O\left(N^{-2}\right)$. 然后利用混合序列的中心极限定理可以 证明 $\sqrt{T / N} \tilde{g}^{V}(u)$ 依分布收玫到正态分布, 均值是 0 、方差是 $V_{u}$. 接下来具体看 $\tilde{g}(u)$ 的收玫速度和渐 近分布. 我们可以证明

$$
\begin{aligned}
\tilde{g}(u)-g(u)= & \boldsymbol{B}(u)^{\mathrm{T}} \tilde{\boldsymbol{\alpha}}-g(u)=\boldsymbol{B}(u)^{\mathrm{T}}\left(\boldsymbol{B}^{\mathrm{T}} \boldsymbol{B}\right)^{-1} \boldsymbol{B}^{\mathrm{T}}\left(\boldsymbol{Y}-\boldsymbol{X}_{\theta_{0}} \tilde{\boldsymbol{\beta}}\right)-g(u) \\
= & \boldsymbol{B}(u)^{\mathrm{T}}\left(\boldsymbol{B}^{\mathrm{T}} \boldsymbol{B}\right)^{-1} \boldsymbol{B}^{\mathrm{T}} \boldsymbol{\varepsilon}+\boldsymbol{B}(u)^{\mathrm{T}}\left(\boldsymbol{B}^{\mathrm{T}} \boldsymbol{B}\right)^{-1} \boldsymbol{B}^{\mathrm{T}} \boldsymbol{g}-g(u) \\
& +\boldsymbol{B}(u)^{\mathrm{T}}\left(\boldsymbol{B}^{\mathrm{T}} \boldsymbol{B}\right)^{-1} \boldsymbol{B}^{\mathrm{T}} \boldsymbol{X}_{\theta_{0}}(\boldsymbol{\beta}-\tilde{\boldsymbol{\beta}}) \\
= & \boldsymbol{B}(u)^{\mathrm{T}}\left(\boldsymbol{B}^{\mathrm{T}} \boldsymbol{B}\right)^{-1} \boldsymbol{B}^{\mathrm{T}} \boldsymbol{\varepsilon}+O\left(N^{-2}\right)+\boldsymbol{B}(u)^{\mathrm{T}}\left(\boldsymbol{B}^{\mathrm{T}} \boldsymbol{B}\right)^{-1} \boldsymbol{B}^{\mathrm{T}}\left(\tilde{\boldsymbol{X}_{\theta_{0}}}+\boldsymbol{e}\right)(\boldsymbol{\beta}-\tilde{\boldsymbol{\beta}}) \\
= & \boldsymbol{B}(u)^{\mathrm{T}}\left(\boldsymbol{B}^{\mathrm{T}} \boldsymbol{B}\right)^{-1} \boldsymbol{B}^{\mathrm{T}} \boldsymbol{\varepsilon}+O\left(N^{-2}\right)+\left(O(1)+\left(\frac{N}{T}\right)^{1 / 2}\right) O_{p}\left(T^{-1 / 2}\right) \\
= & \boldsymbol{B}(u)^{\mathrm{T}}\left(\boldsymbol{B}^{\mathrm{T}} \boldsymbol{B}\right)^{-1} \boldsymbol{B}^{\mathrm{T}} \boldsymbol{\varepsilon}+O\left(N^{-2}\right)+o_{p}(1) .
\end{aligned}
$$

因为

$$
\operatorname{Var}\left(\boldsymbol{B}(u)^{\mathrm{T}}\left(\boldsymbol{B}^{\mathrm{T}} \boldsymbol{B}\right)^{-1} \boldsymbol{B}^{\mathrm{T}} \boldsymbol{\varepsilon}\right)=\boldsymbol{B}(u)^{\mathrm{T}} \boldsymbol{B}^{\mathrm{T}} \boldsymbol{B}^{-1} \operatorname{Var}\left(\boldsymbol{B}^{\mathrm{T}} \boldsymbol{\varepsilon}\right) \boldsymbol{B}^{\mathrm{T}} \boldsymbol{B}^{-1} \boldsymbol{B}(u)=O\left(\left(\frac{N}{T}\right)^{1 / 2}\right)
$$

所以, 如果低平滑条件成立, 即内部节点数满足 $T^{1 / 5}=o(N)$, 那么 $N^{-2}=o\left((N / T)^{1 / 2}\right)$, 偏差相对可以 忽略. 根据混合序列的中心极限定理, 得到

$$
\sqrt{\frac{T}{N}}(\hat{g}(u)-g(u)) \stackrel{d}{\longrightarrow} N\left(0, V_{u}\right)
$$

其中 $V_{u, T}=\boldsymbol{B}(u)^{\mathrm{T}} \Psi^{-1} \Sigma_{2} \Psi^{-1} \boldsymbol{B}(u) / N, \Psi=\frac{\boldsymbol{B}^{\mathrm{T}} \boldsymbol{B}}{T}, \Sigma_{2}=\sum_{i, j=1}^{T} \boldsymbol{B}\left(u_{i}\right) \boldsymbol{B}\left(u_{j}\right)^{\mathrm{T}} E\left(\varepsilon_{i} \varepsilon_{j}\right) / T$.

由于 $\zeta_{0}\left(N_{T}\right)$ 是满足 $\sup _{u \in[0,1]}\|\boldsymbol{B}(u)\| \leqslant \zeta_{0}\left(N_{T}\right)$ 且 $\zeta_{0}\left(N_{T}\right)^{2} N_{T} / T \rightarrow 0$ 的常数数列, 所以一致收 敛速率满足 (参见文献 $[25$, 第 455 页])

$$
\begin{aligned}
\sup _{u \in[0,1]}|\tilde{g}(u)-g(u)|= & \sup _{u \in[0,1]} \mid \boldsymbol{B}(u)^{\mathrm{T}}\left(\boldsymbol{B}^{\mathrm{T}} \boldsymbol{B}\right)^{-1} \boldsymbol{B}^{\mathrm{T}} \boldsymbol{\varepsilon}+\boldsymbol{B}(u)^{\mathrm{T}}\left(\boldsymbol{B}^{\mathrm{T}} \boldsymbol{B}\right)^{-1} \boldsymbol{B}^{\mathrm{T}} \boldsymbol{g}-g(u) \\
& +\boldsymbol{B}(u)^{\mathrm{T}}\left(\boldsymbol{B}^{\mathrm{T}} \boldsymbol{B}\right)^{-1} \boldsymbol{B}^{\mathrm{T}} \boldsymbol{X}_{\theta_{0}}(\boldsymbol{\beta}-\tilde{\boldsymbol{\beta}}) \mid \\
= & O_{p}\left(\zeta_{0}(N)\left(\sqrt{\frac{N}{T}}+N^{-2}\right)\right)
\end{aligned}
$$

定理 3.3 得证. 


\section{Modelling and applications for non-stationary time series in the presence of trend and period}

Shouxia Wang, Jinhong You \& Tao Huang

Abstract In this paper, we study the estimation of the non-stationary time series in the presence of trend and period. We write the classical decomposition time series model as a partial linear model with an unknown parameter. Then we approximate the trend term with B-spline and obtain the estimators of the period, periodic sequence and trend. The asymptotic behaviors of the estimators are given in our paper, including the consistency of period estimation and the asymptotic behavior of the estimated periodic sequence and trend. We illustrate the superiority of our method through simulations and the applications to two real data examples show the utility of our method.

Keywords period estimate, smooth trend, B-spline, penalized least square

MSC(2020) 62G05, 62G08, 62G20, 62P12

doi: $10.1360 /$ SSM-2019-0226 